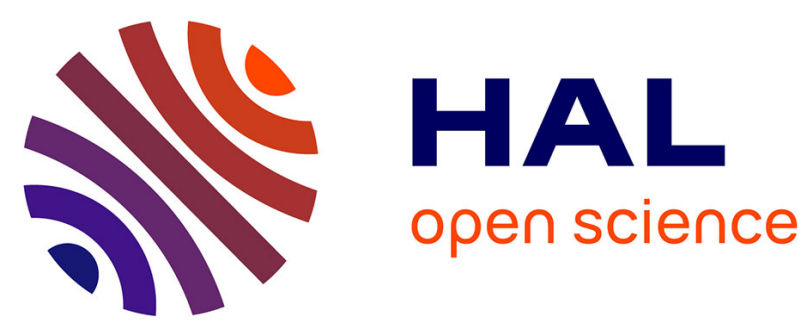

\title{
Surface effects on image stresses and dislocation pile-ups in anisotropic bi-crystals
}

Xiaolei Chen, Thiebaud Richeton, Christian Motz, Stéphane Berbenni

\section{To cite this version:}

Xiaolei Chen, Thiebaud Richeton, Christian Motz, Stéphane Berbenni. Surface effects on image stresses and dislocation pile-ups in anisotropic bi-crystals. International Journal of Plasticity, 2021, pp.102967. 10.1016/j.ijplas.2021.102967 . hal-03157931

\section{HAL Id: hal-03157931 \\ https://hal.univ-lorraine.fr/hal-03157931}

Submitted on 3 Mar 2021

HAL is a multi-disciplinary open access archive for the deposit and dissemination of scientific research documents, whether they are published or not. The documents may come from teaching and research institutions in France or abroad, or from public or private research centers.
L'archive ouverte pluridisciplinaire HAL, est destinée au dépôt et à la diffusion de documents scientifiques de niveau recherche, publiés ou non, émanant des établissements d'enseignement et de recherche français ou étrangers, des laboratoires publics ou privés.

\section{(ㅇ)(1) $\$$}

Distributed under a Creative Commons Attribution - NonCommercial - NoDerivatives 44.0 


\section{Highlights}

- A new bi-crystal surface layer configuration with stiff layer adjacent to the free surface is modeled based on the image decomposition method.

- The numerically computed dislocation image stress is different from purely free or rigid surface.

- Size effects on the dislocation image stress are found in presence of two parallel free surfaces or two grain boundaries.

- The equilibrium dislocation positions are computed for a double-ended dislocation pile-up considering surface conditions, grain boundary and critical stress. 


\title{
Surface effects on image stresses and dislocation pile-ups in anisotropic bi-crystals
}

\author{
Xiaolei Chen ${ }^{\mathrm{a}, \mathrm{b}}$, Thiebaud Richeton ${ }^{\mathrm{a}}$, Christian Motz ${ }^{\mathrm{b}}$, Stéphane Berbenni ${ }^{\mathrm{a}, *}$ \\ ${ }^{a}$ Université de Lorraine, CNRS, Arts et Métiers ParisTech, LEM3, F-57000 Metz, France \\ ${ }^{b}$ Department of Materials Science and Engineering, Saarland University, Saarbrücken, Germany
}

\begin{abstract}
Based on the image decomposition method and the Leknitskii-Eshelby-Stroh (LES) formalism, the effects of surface conditions and misorientation on the dislocation image stress and dislocation distributions in double-ended discrete dislocation pile-ups are studied in a new bi-crystal surface layer configuration. This new configuration contains a stiff layer of finite thickness adjacent to the free surface, which influences the dislocation image stress. The numerical method is first applied to different half-space and bi-crystal configurations for $\mathrm{Ni}$. It is shown that the stiff layer significantly modifies the profile of the dislocation image stress compared to a free or rigid surface. A size effect on the dislocation image stress is found when considering two parallel free surfaces. In addition, the effect of each free surface is individually analyzed. Meanwhile, a grain size effect is also found when considering two parallel grain boundaries. In a second application, the numerical study of a double-ended dislocation pile-up model is proposed, terminating on a grain boundary on one side and on a stiff layer adjacent to a free surface on the other side. The effects of surface conditions, grain boundary and critical stress on dislocation distributions in the
\end{abstract}

\footnotetext{
*Corresponding author: Stéphane Berbenni

Email address: stephane.berbenni@univ-lorraine.fr (Stéphane Berbenni)
} 
double dislocation pile-up are analyzed both at loading and unloading in the case of $\mathrm{Ni}$ bi-crystals.

Keywords:

Bi-crystal, Surface properties, Free surface, Size effects, Double discrete dislocation pile-up, Grain boundary, Anisotropic elasticity

\section{Introduction}

The mechanical properties of finite-sized bi-crystals and polycrystals are dependent on the interaction mechanisms between dislocations and interfaces, such as grain boundaries (GBs) or external surfaces like free surfaces. The interactions between dislocations and GBs/surfaces have many consequences on materials properties and deformation mechanisms. Therefore, the consideration of these complex interactions is important to optimize materials microstructure and their components geometry and size. As typical examples, we can cite the grain size dependence on the yield stress through the Hall-Petch's relationship (Hall, 1951; Petch, 1953; Armstrong, 1970), the strengthening due to grain boundaries (Lim et al., 2016) and twin boundaries (Schneider et al., 2020), the driving force for GB migration (Richeton et al., 2020), interfacial shearing (Chu et al., 2013; Ovid'ko and Sheinerman, 2017), grain rotation (Borodin et al., 2017), slip/twin transmission (Xiao et al., 2017; Lin et al., 2018; Haouala et al., 2020) or the micro-beam size effect due to dislocation starvation at free surfaces (Gao et al., 2010). Indeed, the grain size effect in polycrystalline specimen is usually explained by the stress concentration at the tip of slip bands terminating at GB using dislocation pile-up model (Eshelby et al., 1951; Armstrong, 2014; Cordero et al., 2016). Other explanations can also be invoked considering dislocation emission from GBs and GB dislocation sources (Borovikov et al., 2018; 
Schouwenaars, 2020). Dislocation pile-ups at GBs in polycrystalline materials have been experimentally observed by Transmission Electron Microscopy (TEM) (Shen et al., 1988; Lee et al., 1990; Kondo et al., 2016), Electron Channeling Contrast Imaging (ECCI) (Han et al., 2018; Javaid et al., 2020) and Atomic Force Microscopy (AFM) (Schäfer et al., 2016; Chen et al., 2020; Beucia et al., 2020). Geometrically confined specimen exhibit another type of size effect related to their dimension and free surface effects. For example, polycrystalline freestanding thin film thickness effects have been reported by Espinosa et al. (2004) and Nicola et al. (2006). These plasticity size and surface effects have been numerically interpreted using Discrete Dislocation Dynamics (DDD) (Nicola et al., 2006; Espinosa et al., 2006) or crystal plasticity finite element method (CPFEM) (Keller et al., 2012). Regardless the specific details of all these mechanisms, dislocations always interact with GBs and external surfaces through their elastic fields.

The elastic fields due to three-dimensional dislocation loops in anisotropic multilayered media have been studied using various methods (Han and Ghoniem, 2005; Chu et al., 2012; Chen et al., 2017, 2019b; Yuan et al., 2019). In continuum mechanics, GB effects have been mostly investigated by using a perfect interface model, where the GB is described as a zero-thickness interface between two different anisotropic solids with different crystallographic orientations being perfectly bonded, i.e. satisfying displacement and traction continuities across interface (Stroh, 1958; Suo, 1990; Ting, 1992; Chen et al., 2019a). Similarly, the external surfaces are generally regarded as a surface without thickness and the boundary condition is set to be traction free for the free surface and displacement free for the rigid surface (Suo, 1990; Ting, 1992). However, the external surfaces are rarely purely free or rigid in experiments. Indeed, there is usually a thin layer with different mechanical properties close to the surface due to surface preparations, such as the FIB polishing which can create a damaged 
layer (Kheradmand, 2012; Idrissi et al., 2016; Chen, 2020). In order to study the interactions between dislocations, GBs and surfaces, the elastic fields due to one single dislocation in anisotropic bi-materials and half-spaces have been largely investigated for perfect GB and half-space surface models with two-dimensional elasticity theory (Tucker and Crocker, 1968; Gemperlova and Saxl, 1968; Suo, 1990; Ting, 1992; Belov, 1992; Chu and Pan, 2014; Zhang et al., 2016; Chen et al., 2020; Chen, 2020). In particular, Chen et al. (2019a) recently have studied the interactions between discrete dislocation pile-ups and GB considered as an interphase with finite thickness. The calculations are based on the work of Choi and Earmme (2002a,b) who have derived recurrence formulas to compute the elastic fields associated with dislocations or line forces in the presence of two parallel interfaces/surfaces in both anisotropic and isotropic elasticity. They use an alternating technique to satisfy boundary conditions at both interfaces/surfaces. However, this alternating method is restricted to problems involving two interfaces or two surfaces. For a more general case, such as in multilayered media, Wang et al. (2007) have developed the image decomposition method in two-dimensional anisotropic elasticity with singular Cauchy integral equations based on the continuation method for perfect interfaces and surfaces. This method has no limitation in terms of the number of involved layers, and the interactions between dislocations and an infinite number of parallel interfaces/surfaces can be solved.

Dislocation pile-ups have been theoretically investigated with both continuum and discrete theories (Chou and Li, 1969; Wagoner, 1981; Voskoboinikov et al., 2007; Kochmann and Le, 2008). In a previous study, the Burgers vector distributions in $\mathrm{Ni}$ and $\alpha$-Brass bi-crystalline micropillars have been experimentally characterized by AFM and compared to theoretical calculations considering both GB as an interphase and free surface effects in a single ended dislocation pile-up model (Chen et al., 2020). 
However, the analysis of the slip step height profile in the $\alpha$-Brass sample indicate that the pile-up could actually be double-ended with dislocations also piling-up at an external boundary (Chen et al., 2020). With a free surface, dislocations cannot pile-up at the surface side as they will escape the material due to attractive image force. Thus, a rigid surface or a stiff layer adjacent to the surface is necessary in order to provide a repulsive stress that stabilize the dislocations at the surface side. However, an external purely rigid surface is not realistic. Therefore, it is more reasonable to consider the presence of a stiff layer adjacent to the free surface. Such a configuration may have complex effects on dislocations compared to a free or rigid surface since dislocations interact with more than one surface. Beyond that, a reverse dislocation motion is often observed during the unloading stage of mechanical tests (Kirchlechner et al., 2012; Mompiou et al., 2012). When dislocation pile-ups form, the reverse dislocation motion mainly results from the back-stress induced by the dislocations. Hence, dislocations glide more easily, resulting in a lower yield stress at reverse loading which is called the Bauschinger effect (Dieter, 1986). This Bauschinger effect has been experimentally observed in micro-compression tests (Pedersen et al., 1981; Suresh, 1998; Rajagopalan et al., 2010) and theoretically studied using DDD (Davoudi et al., 2014), fast Fourier transform-based mesoscale field dislocation mechanics (MFDM) in polycrystals (Berbenni et al., 2020) or by strain gradient plasticity (SGP) models (Niordson and Legarth, 2010; Wulfinghoff et al., 2015; Rys and Petryk, 2018; Voyiadjis and Song, 2019; Jebahi et al., 2020).

In the present study, the objective is to theoretically investigate the combined effects of external surface and GB on the image stress of single dislocations and on the equilibrium configurations of double-ended dislocation pile-ups in anisotropic elasticity. Thus, different bi-crystal configurations are explored by using the image decomposition method for heterogeneous anisotropic multilayer configurations. In 
contrast with our previous studies (Chen et al., 2019a, 2020), the thickness of the bi-crystal is here finite and the presence of stiff layers adjacent to external free surfaces is considered. In section 2, the image decomposition method of Wang et al. (2007) is firstly summarized for a problem including three layers. In particular, the full components of all the matrices involved in the final solution are provided in Appendix C. It should be pointed out that this method is not further developed in the present paper. Here, the method is applied for studying the effects of different surface configurations on a single dislocation in section 3.1. A new configuration called bi-crystal surface layer configuration with a stiff layer adjacent to the free surface is proposed. The effects of such a configuration on the dislocation image stress are investigated by comparisons with completely free or rigid surfaces. In addition, a size effect on the dislocation image stress is found when considering two parallel free surfaces or two parallel GBs . A second application of the image decomposition method is proposed in section 3.2 for double-ended dislocation pile-ups. A doubleended dislocation pile-up model with a Frank-Read dislocation source mechanism is proposed considering loading/unloading processes and the effect of the critical stress. The double-ended pile-up terminates on GB on one side and on a stiff layer adjacent to a free surface on the other side. Dislocation distributions are thus studied for different surface configurations and critical stress values.

\section{Image decomposition method for elastic fields of dislocations in anisotropic multilayered materials}

In order to better understand the image decomposition method developed by Wang et al. (Wang et al., 2007), the details of this method are first summarized for a tri-material configuration with two free surfaces. However, it should be pointed

out that within this method, the number of layers is unlimited, see e.g. the four 
layers configuration described in Figure 3 (b). All the notation conventions used in this section and their definitions are listed in Appendix A.

\subsection{Multilayered material configuration}

Figure 1 shows a tri-material configuration with two free surfaces. There are four planar faces whose normals are directed along the $x_{2}$-axis. These faces are located at $x_{2}=0, x_{2}=-h_{1}, x_{2}=-\left(h_{1}+h_{2}\right)$ and $x_{2}=-\left(h_{1}+h_{2}+h_{3}\right)$ for top free surface, first interface, second interface and bottom free surface, respectively. Material I corresponds to $-h_{1}<x_{2}<0$, material II to $-\left(h_{1}+h_{2}\right)<x_{2}<-h_{1}$ and material III to $-\left(h_{1}+h_{2}+h_{3}\right)<x_{2}<-\left(h_{1}+h_{2}\right)$. Thus, the thickness of the whole material is marked as $H^{\prime}=h_{1}+h_{2}+h_{3}$. All the materials are assumed to be perfectly bonded to each other at interfaces.

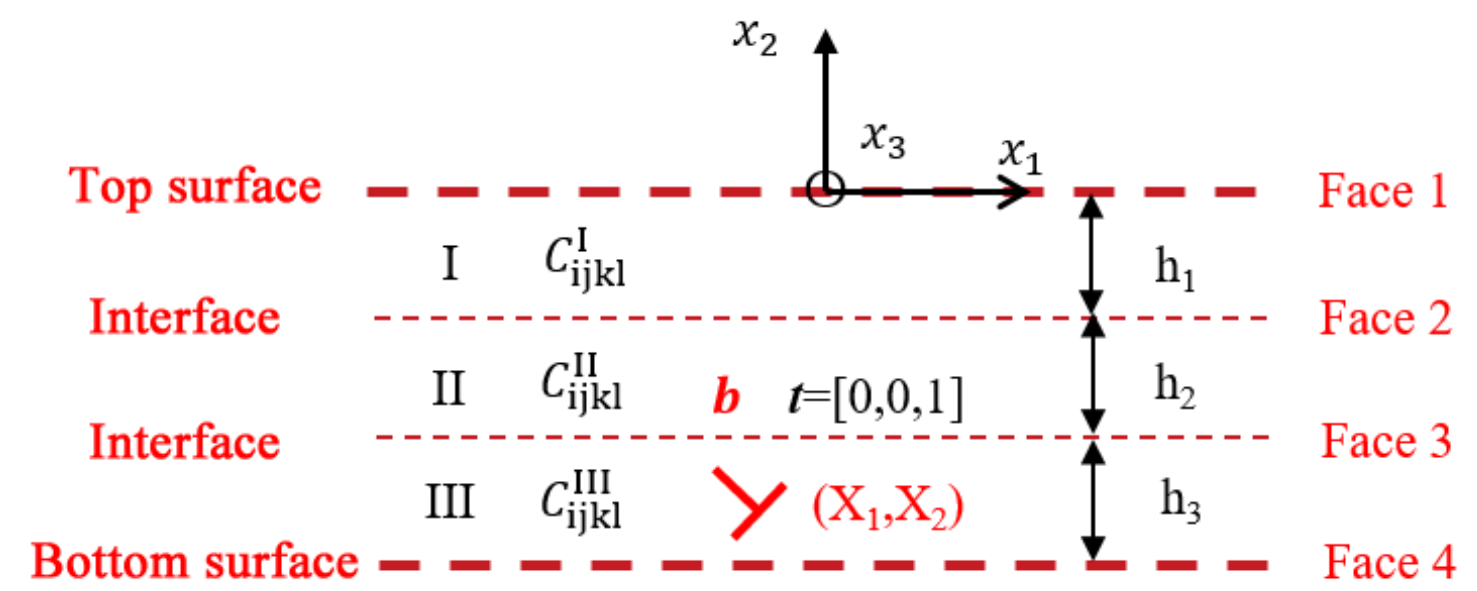

Figure 1: Schematic figure of one single infinite straight dislocation in a tri-material configuration with three different stiffness tensors $C_{i j k l}^{\mathrm{I}}, C_{i j k l}^{\mathrm{II}}$ and $C_{i j k l}^{\mathrm{III}}$ for material I, II and III, respectively. There are two planar free surfaces: the top free surface (Face 1) and the bottom free surface (Face 4) and two perfectly bonded interfaces (Face 2 and Face 3 ). 


\subsection{Image decomposition method}

The principle of the image decomposition method proposed by Wang et al. (2007) is to decompose the problem into several subproblems of infinite homogeneous medium for each material. Two types of image dislocation densities are distributed on each interface while one image dislocation density is distributed on each surface as shown in Figure 2 (left). The densities of these image dislocations are denoted $\rho^{t}$, where $t=1,2$ and 3 indicate the $x_{1}-, x_{2}-$ and $x_{3}$-components, respectively. Thus, in the 3-layers configuration of Figure 1, there are a total of 18 image dislocation densities $\rho_{1 U}^{t}, \rho_{1 L}^{t}, \rho_{2 U}^{t}, \rho_{2 L}^{t}, \rho_{3 U}^{t}$ and $\rho_{3 L}^{t}$, which are located at $x_{2}=0^{-},-h_{1}^{+},-h_{1}^{-},-\left(h_{1}+h_{2}\right)^{+},-\left(h_{1}+h_{2}\right)^{-}$and $-\left(h_{1}+h_{2}+h_{3}\right)^{+}$, respectively.

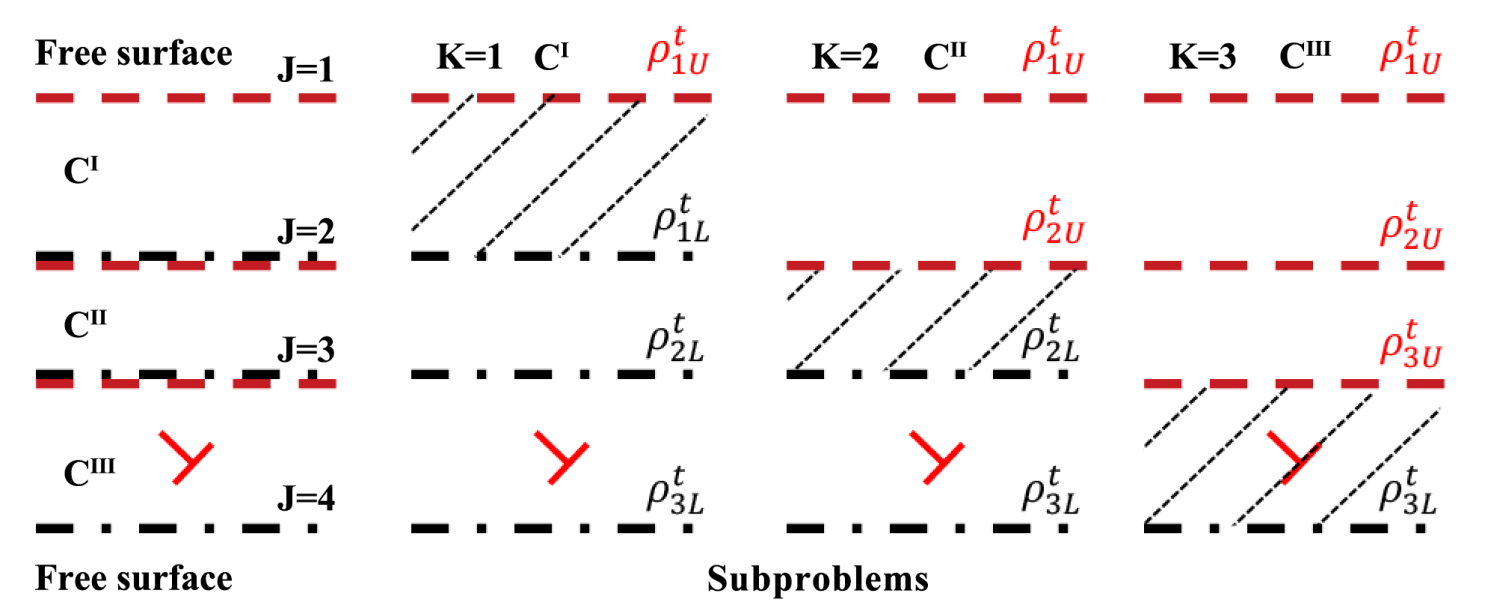

Figure 2: Decomposition of a 3-layers configuration (left) with two free surfaces and two interfaces into three infinite homogeneous subproblems. The position of the single dislocation is marked with a red edge dislocation symbol.

These densities can be resolved at the same time by simultaneously considering all the 18 boundary conditions as follows $(i=1,2$ and 3$)$ :

- The tractions $\sigma_{i 2}$ vanish on both free surfaces: 
I. $\sigma_{i 2}\left(x_{1}, x_{2}=0\right)=0$

II. $\sigma_{i 2}\left(x_{1}, x_{2}=-\left(h_{1}+h_{2}+h_{3}\right)\right)=0$

- The continuities of stresses $\sigma_{i 2}$ and tangential displacement gradients $\partial u_{i} / \partial x_{1}$ across both interfaces:

III. $\sigma_{i 2}\left(x_{1}, x_{2}=-h_{1}^{+}\right)=\sigma_{i 2}\left(x_{1}, x_{2}=-h_{1}^{-}\right)$

IV. $\frac{\partial u_{i}}{\partial x_{1}}\left(x_{1}, x_{2}=-h_{1}^{+}\right)=\frac{\partial u_{i}}{\partial x_{1}}\left(x_{1}, x_{2}=-h_{1}^{-}\right)$

V. $\sigma_{i 2}\left(x_{1}, x_{2}=-\left(h_{1}+h_{2}\right)^{+}\right)=\sigma_{i 2}\left(x_{1}, x_{2}=-\left(h_{1}+h_{2}\right)^{-}\right)$

VI. $\frac{\partial u_{i}}{\partial x_{1}}\left(x_{1}, x_{2}=-\left(h_{1}+h_{2}\right)^{+}\right)=\frac{\partial u_{i}}{\partial x_{1}}\left(x_{1}, x_{2}=-\left(h_{1}+h_{2}\right)^{-}\right)$

The elastic fields in each layer are determined by summing the contributions of the single dislocation and all the corresponding image dislocations as shown in Figure 2 (right). Following Wang et al. (2007)), the image dislocation densities in the three subproblems are $\rho_{J 1}^{t}=\left\{\rho_{1 U}^{t}, \rho_{1 L}^{t}, \rho_{2 L}^{t}, \rho_{3 L}^{t}\right\}$ for the first subproblem, $\rho_{J 2}^{t}=$ $\left\{\rho_{1 U}^{t}, \rho_{2 U}^{t}, \rho_{2 L}^{t}, \rho_{3 L}^{t}\right\}$ for the second one and $\rho_{J 3}^{t}=\left\{\rho_{1 U}^{t}, \rho_{2 U}^{t}, \rho_{3 U}^{t}, \rho_{3 L}^{t}\right\}$ for the third one as shown in Figure 2, where the subscript $J$ is the index of the image dislocation densities for each subproblem. $J=1: 4$ for a configuration with 3 layers.

\subsection{Governing integral equations}

Based on the Leknitskii-Eshelby-Stroh (LES) formalism (Leknitskii, 1963; Eshelby et al., 1953; Stroh, 1958), the stress and displacement gradient fields due to a mixed dislocation with Burgers vector components $b_{t}(t=1,2,3)$ located at $\left(x_{1 s}, x_{2 s}\right)$ in a homogeneous anisotropic medium under plane strain conditions are (Anderson et al., 1968; Wang et al., 2007): 


$$
\begin{aligned}
& \sigma_{i j}\left(K, b_{t}, \Delta x_{1}, \Delta x_{2}\right)= \\
& 2\left[\xi_{K \underline{\alpha} i j}^{t R} P\left(\Delta x_{1}, p_{K \underline{\alpha}}^{R} \Delta x_{2}, p_{K \underline{\alpha}}^{I} \Delta x_{2}\right)+\xi_{K \underline{\alpha} i j}^{t I} Q\left(\Delta x_{1}, p_{K \underline{\alpha}}^{R} \Delta x_{2}, p_{K \underline{\alpha}}^{I} \Delta x_{2}\right)\right] b_{t} \\
& \frac{\partial u_{i}}{\partial x_{j}}\left(K, b_{t}, \Delta x_{1}, \Delta x_{2}\right)= \\
& 2\left[\zeta_{K \underline{\alpha} i j}^{t R} P\left(\Delta x_{1}, p_{K \underline{\alpha}}^{R} \Delta x_{2}, p_{K \underline{\alpha}}^{I} \Delta x_{2}\right)+\zeta_{K \underline{\alpha} i j}^{t I} Q\left(\Delta x_{1}, p_{K \underline{\alpha}}^{R} \Delta x_{2}, p_{K \underline{\alpha}}^{I} \Delta x_{2}\right)\right] b_{t}
\end{aligned}
$$

where $\Delta x_{1}=x_{1}-x_{1 s}$ and $\Delta x_{2}=x_{2}-x_{2 s}$. The superscripts $R$ and $I$ indicate the real and imaginary parts of each term, respectively. The subscript $K$ indicates which subproblem is considered (no sum over $K$ ). The matrices $\xi_{K \alpha i j}^{t}, \zeta_{K \alpha i j}^{t}$ and the functions $P, Q$ are given by (Wang and $\mathrm{Wu}, 2005$ ):

$$
\begin{aligned}
& \left\{\begin{aligned}
\xi_{K \alpha i j}^{t} & =\frac{C_{K i j \underline{k l}}\left(\delta_{\underline{l} 1}+p_{K \alpha} \delta_{\underline{l} 2}\right) A_{K \underline{k} \alpha} B_{K t \alpha}}{2 \pi \mathrm{i}} \\
\zeta_{K \alpha i j}^{t} & =\frac{\left(\delta_{j 1}+p_{K \alpha} \delta_{j 2}\right) A_{K i \alpha} B_{K t \alpha}}{2 \pi \mathrm{i}}
\end{aligned}\right. \\
& \left\{\begin{array}{l}
P\left(x_{1}-x_{1 s}, a, b\right)=\frac{x_{1}-x_{1 s}+a}{\left(x_{1}-x_{1 s}+a\right)^{2}+b^{2}} \\
Q\left(x_{1}-x_{1 s}, a, b\right)=\frac{b}{\left(x_{1}-x_{1 s}+a\right)^{2}+b^{2}}
\end{array}\right.
\end{aligned}
$$

where $\mathrm{i}$ (non-italicized) is the imaginary unit $\sqrt{-1}, p_{\alpha}$ and $A_{k \alpha}$ with $\alpha=1,2$ and 3 denote the eigenvalues with positive imaginary parts and the associated eigenvectors of the Stroh sextic equation and $B_{t \alpha}=\left(C_{t 2 \underline{k} 1}+p_{\alpha} C_{t 2 \underline{k} 2}\right) A_{\underline{k} \alpha}$ (Stroh, 1958; Suo, 1990).

The contribution of image dislocations densities on elastic fields can be calculated by replacing the Burgers vector $b_{t}$ by $\rho_{J K}^{t}\left(x_{1}^{\prime}\right)$ and integrating from $x_{1}^{\prime}=-\infty$ to $x_{1}^{\prime}=+\infty$ in Eqs. 1 and 2 (Wang et al., 2007): 


$$
\begin{aligned}
& \sigma_{i j}\left(K, \rho_{J K}^{t}, \Delta x_{1}, \Delta x_{2}\right)= \\
& \int_{-\infty}^{+\infty} 2\left[\xi_{K \underline{\alpha} i j}^{t R} P\left(\Delta x_{1}, p_{K \underline{\alpha}}^{R} \Delta x_{2}, p_{K \underline{\alpha}}^{I} \Delta x_{2}\right)+\xi_{K \underline{\alpha} i j}^{t I} Q\left(\Delta x_{1}, p_{K \underline{\alpha}}^{R} \Delta x_{2}, p_{K \underline{\alpha}}^{I} \Delta x_{2}\right)\right] \rho_{J K}^{t}\left(x_{1}^{\prime}\right) d x_{1}^{\prime} \\
& =2 \pi\left[\xi_{K \underline{\alpha} i j}^{t R} H\left(p_{K \underline{\alpha}}^{R} \Delta x_{2}, p_{K \underline{\alpha}}^{I} \Delta x_{2}\right)+\xi_{K \underline{\alpha} i j}^{t I} I\left(p_{K \underline{\alpha}}^{R} \Delta x_{2}, p_{K \underline{\alpha}}^{I} \Delta x_{2}\right)\right]\left[\rho_{J K}^{t}\left(x_{1}\right)\right]
\end{aligned}
$$

$$
\begin{aligned}
& \frac{\partial u_{i}}{\partial x_{j}}\left(K, \rho_{J K}^{t}, \Delta x_{1}, \Delta x_{2}\right)= \\
& \int_{-\infty}^{+\infty} 2\left[\zeta_{K \underline{\alpha} i j}^{t R} P\left(\Delta x_{1}, p_{K \underline{\alpha}}^{R} \Delta x_{2}, p_{K \underline{\alpha}}^{I} \Delta x_{2}\right)+\zeta_{K \underline{\alpha} i j}^{t I} Q\left(\Delta x_{1}, p_{K \underline{\alpha}}^{R} \Delta x_{2}, p_{K \underline{\alpha}}^{I} \Delta x_{2}\right)\right] \rho_{J K}^{t}\left(x_{1}^{\prime}\right) d x_{1}^{\prime} \\
& =2 \pi\left[\zeta_{K \underline{\alpha} i j}^{t R} H\left(p_{K \underline{\alpha}}^{R} \Delta x_{2}, p_{K \underline{\alpha}}^{I} \Delta x_{2}\right)+\zeta_{K \underline{\alpha} i j}^{t I} I\left(p_{K \underline{\alpha}}^{R} \Delta x_{2}, p_{K \underline{\alpha}}^{I} \Delta x_{2}\right)\right]\left[\rho_{J K}^{t}\left(x_{1}\right)\right]
\end{aligned}
$$

where $\Delta x_{1}=x_{1}-x_{1}^{\prime}, \Delta x_{2}=x_{2}-x_{2}^{\prime}$ and $\left(x_{1}^{\prime}, x_{2}^{\prime}\right)$ denote the positions of image dislocation densities. The definitions of the integral transforms $H$ and $I$ are (Wang et al., 2007):

$$
\left\{\begin{aligned}
H(a, b)\left[\rho\left(x_{1}\right)\right] & =\frac{1}{\pi} \int_{-\infty}^{\infty} P\left(x_{1}-x_{1}^{\prime}, a, b\right) \rho\left(x_{1}^{\prime}\right) d x_{1}^{\prime} \\
I(a, b)\left[\rho\left(x_{1}\right)\right] & =\frac{1}{\pi} \int_{-\infty}^{\infty} Q\left(x_{1}-x_{1}^{\prime}, a, b\right) \rho\left(x_{1}^{\prime}\right) d x_{1}^{\prime}
\end{aligned}\right.
$$

Combining Eqs. 1 - 7, the boundary conditions can be derived with respect to the Burgers vector of the single dislocation and image dislocations densities. For example, the boundary condition I now writes: 


$$
\begin{aligned}
& \sigma_{i 2}\left(1, b_{t}, x_{1}-x_{1 s}, x_{2}-x_{2 s}\right)+\sum_{J=1}^{J=4} \sigma_{i 2}\left(1, \rho_{J 1}^{t}, x_{1}-x_{1}^{\prime}, x_{2}-x_{2 J}\right)= \\
& 2\left[\xi_{1 \underline{t} i 2}^{\underline{i} R} P\left(x_{1}-x_{1 s},-p_{1 \underline{\alpha}}^{R} x_{2 s},-p_{1 \underline{\alpha}}^{I} x_{2 s}\right)+\xi_{1 \underline{t} \underline{t} i 2}^{\underline{I}} Q\left(x_{1}-x_{1 s},-p_{1 \underline{\alpha}}^{R} x_{2 s},-p_{1 \underline{\alpha}}^{I} x_{2 s}\right)\right] b_{\underline{t}} \\
& +2 \pi\left[\xi_{1 \underline{\alpha} i 2}^{\underline{t} R} H(0,0)+\xi_{1 \underline{t} \underline{t} i 2}^{\underline{I}} I\left(0,0^{-}\right)\right]\left[\rho_{1 U}^{\underline{t}}\left(x_{1}\right)\right] \\
& +2 \pi\left[\xi_{1 \underline{\underline{\alpha} i 2} 2}^{\underline{t}} H\left(p_{1 \underline{\alpha}}^{R} h_{1}, p_{1 \underline{\alpha}}^{I} h_{1}\right)+\xi_{1 \underline{\alpha} i 2}^{\underline{I} I} I\left(p_{1 \underline{\alpha}}^{R} h_{1}, p_{1 \underline{\alpha}}^{I} h_{1}\right)\right]\left[\rho_{1 L}^{t}\left(x_{1}\right)\right] \\
& +2 \pi\left[\xi_{\underline{1} \underline{\underline{\alpha} i 2}}^{\underline{t} R} H\left(p_{1 \underline{\underline{\alpha}}}^{R}\left(h_{1}+h_{2}\right), p_{1 \underline{\alpha}}^{I}\left(h_{1}+h_{2}\right)\right)+\right. \\
& \left.\xi_{1 \underline{\underline{\underline{\alpha}} i 2}}^{\underline{\underline{\alpha}} I} I\left(p_{1 \underline{\underline{\alpha}}}^{R}\left(h_{1}+h_{2}\right), p_{1 \underline{\underline{\alpha}}}^{I}\left(h_{1}+h_{2}\right)\right)\right]\left[\rho_{2 L}^{\underline{t}}\left(x_{1}\right)\right] \\
& +2 \pi\left[\xi_{\underline{1} \underline{\underline{\alpha} i 2}}^{\underline{R}} H\left(p_{1 \underline{\alpha}}^{R}\left(h_{1}+h_{2}+h_{3}\right), p_{1 \underline{\alpha}}^{I}\left(h_{1}+h_{2}+h_{3}\right)\right)+\right. \\
& \left.\xi_{1 \underline{\underline{t} i 2}}^{\underline{I} I} I\left(p_{1 \underline{\underline{\alpha}}}^{R}\left(h_{1}+h_{2}+h_{3}\right), p_{1 \underline{\alpha}}^{I}\left(h_{1}+h_{2}+h_{3}\right)\right)\right]\left[\rho_{3 L}^{\underline{t}}\left(x_{1}\right)\right]=0
\end{aligned}
$$

where $\left(x_{1}^{\prime}, x_{2 J}\right)$ are the coordinates of image dislocation densities $\rho_{J K}^{t}$ for the subproblem $K$.

In Eq. 8, a singularity term is found when the integral transform $H(0,0)$ directly operates on the image dislocation density $\boldsymbol{\rho}_{1 U}\left(x_{1}\right)$. This singularity problem has been solved by Wang et al. (2007). Their mathematical method is summarized in Appendix B. After removing the singularity terms, a system of nonlinear equations with 18 Fredholm equations of second kind in terms of the image dislocation densities $\boldsymbol{\rho}$ (Eq. B.10) is obtained by considering all the 18 boundary conditions I-VI. These image dislocation densities can be numerically resolved by using the Nyström method (Press et al., 2007). Essentially, the integrals over infinite space can be approximated thanks to a Gauss-Hermite quadrature method. After substituting the integral terms, approximate analytical expressions of $\boldsymbol{\rho}$ can be obtained as a function of $x_{1}$. Finally, the elastic fields, such as the stress fields due to one single dislocation in a 3-layers anisotropic material at any point can be calculated using Eqs. 1-5. 
In the present paper, this numerical method is implemented for different multilayer configurations in order to study the effects of free surfaces and stiff layers as shown in Figure 3. The verification of the correctness of the numerical implementation is performed by comparing the results of image resolved shear stress in tri-crystal configurations with those obtained by the alternating technique of Choi and Earmme (2002a), see Figure 9 in section 3.1. As it can be observed, the agreement between both methods is excellent. For the forthcoming results interpretation, it is recalled that dislocations are considered as infinite straight lines parallel to perfect and planar interfaces and that the properties of dislocation cores are not modeled (linear elasticity). Also, the interactions between two non-parallel dislocations cannot be considered within this two-dimensional framework. Besides, regarding comparisons with experiments, the reader is referred to a recent paper by the same authors (Chen et al., 2020), where Burgers vector distributions of double-ended dislocation pile-ups terminating at GB have been measured by AFM in Ni and $\alpha$-Brass micro-pillars and compared to micromechanical calculations based on the present image decomposition method.

\section{Applications and discussion}

3.1. Coupled effects of surface conditions and misorientation on image stress for a single dislocation

\subsubsection{Surface conditions in half-space and bi-crystal configurations}

The image decomposition method is firstly used to study the effects of different external surface conditions on the elastic fields and especially on image stresses. First of all, the surface conditions in a half-space are shown in Figure 3 (a). The external surface is located at $x_{2}=0$ as marked by surface $\Lambda$. The crystal is semi-infinite 
and occupies the space $x_{2}>0$. In a simple approach, a surface can be typically considered as free or rigid. A free surface has an attractive effect on dislocations whereas a rigid one has a repulsive effect (Ting, 1992; Chen et al., 2019a). However, in experiments, the surface is hardly ideally free or rigid. There is usually a thin layer with different mechanical properties close to the surface due to surface preparation. For example, FIB polishing creates a damaged layer as shown and discussed by Kheradmand (2012), Idrissi et al. (2016) and Chen et al. (2020). In that case, the crystal structure of the damaged layer may change dramatically and may become amorphous. Even the chemical composition may be different. Therefore, we propose here a new half-space surface layer configuration, where the surface can be regarded as an isotropic stiff layer in addition to the external free surface $\Lambda$ as shown in Figure 3 (a). The thickness of this surface layer is denoted $h_{\text {Layer }}$ and it has thus a different elastic stiffness tensor $\mathrm{C}_{\mathrm{ijkl}}^{\mathrm{Layer}}$ compared to the bulk crystal. In order to reflect a different stiffness, $\mathrm{C}_{\mathrm{ijkl}}^{\text {Layer }}$ can be determined by:

$$
\mathrm{C}_{\mathrm{ijkl}}^{\text {Layer }}=\lambda \mathrm{C}_{\mathrm{ijkl}}^{\mathrm{Iso}}
$$

where $\lambda$ is a scalar stiffness parameter and $\mathrm{C}_{\mathrm{ijkl}}^{\mathrm{Iso}}$ is the isotropic elastic stiffness tensor of the material. It should be pointed out that the elastic stiffness tensor of this surface layer is certainly anisotropic, but it remains difficult to have an exact knowledge of this fourth order tensor. As the main objective is to focus on the stiffness effect of the surface layer on the dislocation image stress, the surface layer elastic stiffness tensor $\mathrm{C}_{\mathrm{ijkl}}^{\mathrm{Layer}}$ is simply modelled as isotropic for the studied material and the magnitude of the stiffness is parametrized by a single scalar parameter $\lambda$. Hence, a stiff layer is considered when $\lambda>1$, whereas a compliant layer is reflected by $\lambda<1$.

Second, a new bi-crystal surface layer configuration with one GB and stiff layers 
as shown in Figure 3 (b) is considered. The GB is regarded as a perfectly bonded planar interface (with zero thickness) to only focus the discussion on the respective effects of surface conditions and misorientation. The thicknesses of crystals I and II are marked as $h$ as shown in Figure 3 (b-c).

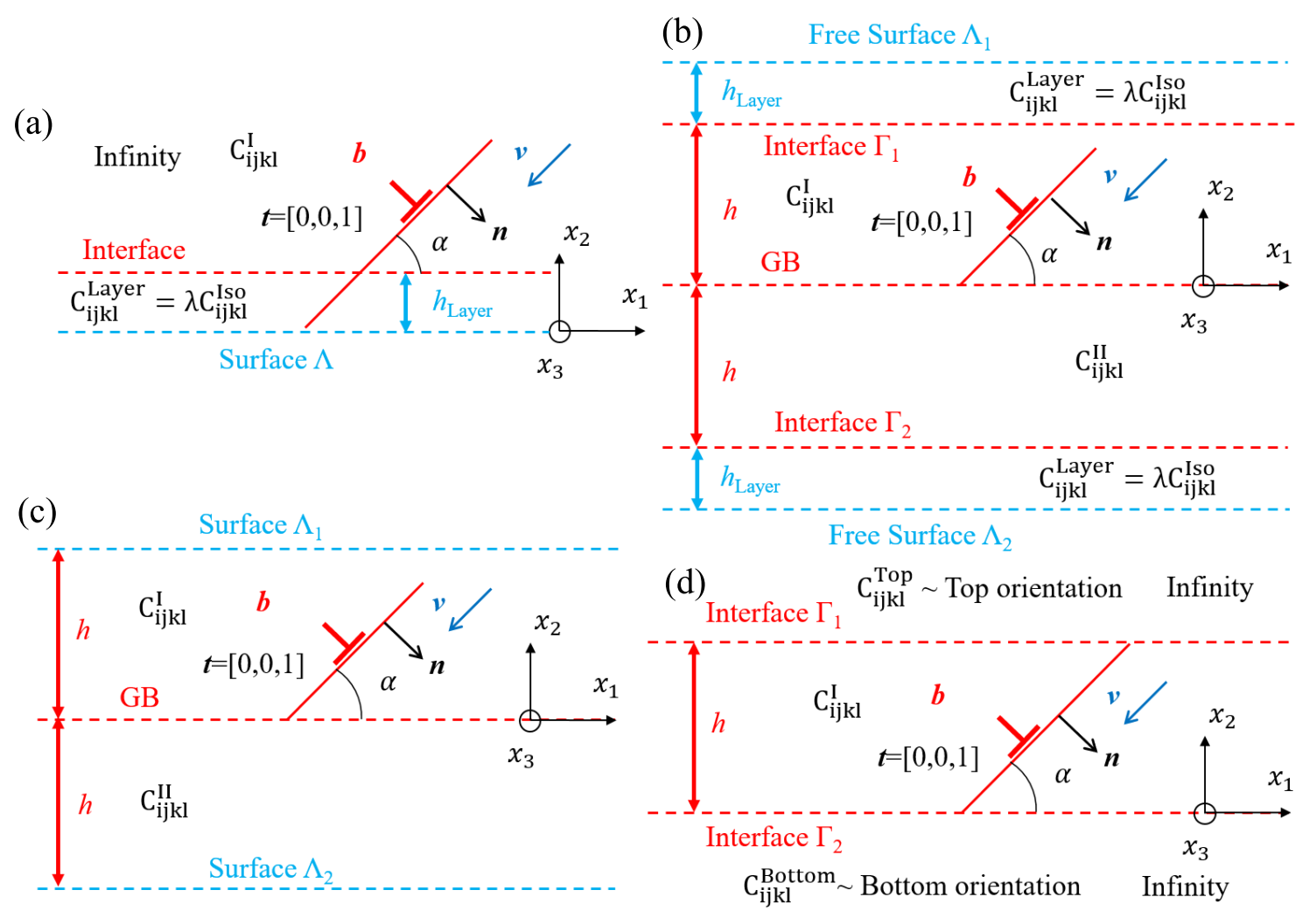

Figure 3: Schematic figure of one single dislocation in different configurations: (a) Half-space with different surface configurations, (b) Bi-crystal surface layer configuration with perfectly planar GB and stiff layers close to free surfaces, (c) Bi-crystal free surface configuration with perfectly planar GB and free surfaces and (d) Infinite tri-crystal configuration with two perfectly planar GBs. In order to well investigate the effects of surface conditions and misorientations, for the bi-crystal configurations the thicknesses of the two crystals and the two surface layers are always the same, which are marked as $h$ and $h_{\text {Layer }}$, respectively. The notations $\boldsymbol{t}, \boldsymbol{b}$ and $\boldsymbol{v}$ indicate the dislocation line vector, Burgers vector and dislocation glide direction, respectively. 
Third, in order to study possible finite size effects on dislocation image stresses, the effects of simple free surfaces are also investigated in the bi-crystal free surface configuration as shown in Figure 3 (c). From Figure 3 (c), four types of bi-crystal configurations with different surface conditions are actually considered in order to assess the effects of each free surface associated with misorientation effects: infinite bi-crystal without surfaces $\Lambda_{1}$ and $\Lambda_{2}$ (crystals I and II are infinite), top free surface with only surface $\Lambda_{1}$ but without $\Lambda_{2}$ (crystal II is infinite), bottom free surface with only surface $\Lambda_{2}$ but without $\Lambda_{1}$ (crystal I is infinite) and two free surfaces with both surfaces $\Lambda_{1}$ and $\Lambda_{2}$.

Finally, an infinite tri-crystal configuration where the crystal of thickness $h$ is sandwiched between two planar GBs is considered as shown in Figure 3 (d). This last configuration can be used to study a possible grain size effect on dislocation image stresses. The dislocation is located in the middle crystal of the tri-crystal and interacts with both GBs. Both top and bottom crystals are infinite media with given orientations.

For clarity, the notations and the definitions of all these configurations are summarized in Table 1. 


\begin{tabular}{ll}
\hline Half-space surface layer configuration & $\begin{array}{l}\text { The surface condition of half-space is a stiff layer defined } \\
\text { by } \lambda \text { and } h_{\text {Layer }} \text { with a external free surface } \Lambda \text { as shown in } \\
\end{array}$ \\
\hline Figure 3 (a)
\end{tabular}

Table 1: Notations and definitions of the different studied configurations.

\subsubsection{Material parameters for image stress calculations}

For all the following numerical applications, the considered material is nickel (Ni) which has a face centered cubic (FCC) structure with cubic elastic stiffness constants $C_{11}=246.5 \mathrm{GPa}, C_{12}=147.3 \mathrm{GPa}$ and $C_{44}=124.7 \mathrm{GPa}$ (Anderson 
et al., 1968). Thus, it has an elastic anisotropy ratio (Zener coefficient) of $A_{\text {ani }}=$ $2 C_{44} /\left(C_{11}-C_{12}\right)=2.51$. In the case of elastic isotropy $\left(A_{\text {ani }}=1\right)$, the considered elastic constants are $C_{11}=306.1 \mathrm{GPa}, C_{12}=116.7 \mathrm{GPa}$ and $C_{44}=94.7 \mathrm{GPa}$ which are deduced from the anisotropic elastic stiffness moduli by applying the Voigt-ReussHill average (Hill, 1952; Dewit, 2008). The lattice parameter of $\mathrm{Ni}$ is $a=0.3524 \mathrm{~nm}$ and thus the length of its standard Burgers vector is $|\boldsymbol{b}|=\frac{\sqrt{2}}{2} \times a=0.2492 \mathrm{~nm}$. The crystallographic orientation of the crystal containing the dislocation is determined from the choice of the active slip system (Chen et al., 2019a). In the global simulation frame $\left(x_{1}, x_{2}, x_{3}\right)$ as shown in Figure 3, the slip system is determined by the Burgers vector $\boldsymbol{b}$ and the slip plane unit normal vector $\boldsymbol{n}=\boldsymbol{t} \times \boldsymbol{m}$ following the Finish Start/Right Hand (FS-RH) convention for a dislocation with a non zero edge component, where $\boldsymbol{t}=[0,0,1]$ is the dislocation line vector and $\boldsymbol{m}=\boldsymbol{b} /|\boldsymbol{b}|$. At the same time, the slip system is supposed to be one of the usual twelve slip systems for FCC crystals, such that $\boldsymbol{m}^{0}=1 / \sqrt{2}[1,1,0]$ and $\boldsymbol{n}^{0}=1 / \sqrt{3}[-1,1,-1]$ in the crystal's frame $\left(e_{1}, e_{2}, e_{3}\right)$. Then the crystallographic orientation of the crystal containing the dislocation is defined by the transformation matrix $\boldsymbol{T}$ such that $[\boldsymbol{m}, \boldsymbol{n}, \boldsymbol{t}]^{\mathrm{T}}=\boldsymbol{T}\left[\boldsymbol{m}^{0}, \boldsymbol{n}^{0}, \boldsymbol{t}^{0}\right]^{\mathrm{T}}$, where $\boldsymbol{t}^{0}=\boldsymbol{m}^{0} \times \boldsymbol{n}^{0}$. For the numerical applications in subsections 3.1.4-3.1.5, only edge dislocations with Burgers vector $\boldsymbol{b}$ in the $\left(x_{1}, x_{2}\right)$ plane are considered. The angle $\alpha$ defined in Figure 3 is set to $\alpha=45^{\circ}$, so that $\boldsymbol{m}=\left[-\frac{\sqrt{2}}{2},-\frac{\sqrt{2}}{2}, 0\right]$. The crystallographic orientation of the bottom crystal can be arbitrarily fixed as there is no dislocation in it. Here, two specific orientations are considered as in Chen et al. (2019a): orientation $\mathrm{A}^{\text {Ori }}$ with $x_{1}=e_{1}, x_{2}=e_{2}$, $x_{3}=e_{3}$ and orientation $\mathrm{B}^{\text {Ori }}$ with $x_{1}=-\frac{1}{2} e_{1}+\frac{\sqrt{2}}{2} e_{2}+\frac{1}{2} e_{3}, x_{2}=-\frac{1}{2} e_{1}-\frac{\sqrt{2}}{2} e_{2}+\frac{1}{2} e_{3}$, $x_{3}=\frac{\sqrt{2}}{2} e_{1}+\frac{\sqrt{2}}{2} e_{3}$. The orientations $\mathrm{A}^{\text {Ori }}$ and $\mathrm{B}^{\text {Ori }}$ have a misorientation angle $35.3^{\circ}$ and $54.7^{\circ}$ with the crystal containing the dislocation, respectively. 


\subsubsection{Image force and image stress}

For the analysis of image force calculations on a dislocation, the projection of the image force along the glide direction $\boldsymbol{v}$ is considered:

$$
f_{\mathrm{im}}=\left[\left(\boldsymbol{\sigma}_{\mathrm{int}} \cdot \boldsymbol{b}\right) \times \boldsymbol{t}\right] \cdot \boldsymbol{v}
$$

For these calculations, the glide direction is set to $\boldsymbol{v}=[-\cos \alpha,-\sin \alpha, 0]$. Hence, $\boldsymbol{v}$ points towards the surface in a half-space configuration (Figure 3 (a)) or the GB in a bi-crystal configuration (Figures $3(\mathrm{~b}-\mathrm{c})$ ) or the lower GB in an infinite tri-crystal configuration with two GBs (Figure $3(\mathrm{~d})$ ). In the present paper, this projected image force is used to compute the image resolved shear stress on a dislocation by dividing by the corresponding Burgers vector magnitude as below:

$$
\tau_{\mathrm{im}}=f_{\mathrm{im}} /|\boldsymbol{b}|
$$

Therefore, $\tau_{\mathrm{im}}>0$ means the GB or the surface attracts the dislocation whereas $\tau_{\mathrm{im}}<0$ means that the dislocation is repelled by the GB or the surface. In particular, it has been shown that orientation $\mathrm{A}^{\text {Ori }}$ has an attractive effect on the edge dislocation defined by $\alpha=45^{\circ}$ while orientation $\mathrm{B}^{\text {Ori }}$ provides a repulsive effect on this edge dislocation (Chen et al., 2019a). Furthermore, it should be pointed out that for the forthcoming results (see subsections 3.1.4-3.1.8), the distance from GB is the distance along the GB normal direction.

\subsubsection{Half-space surface layer configuration: effect of different surface conditions on dislocation image resolved shear stress}

Figure 4 shows the variation of the image resolved shear stress $\tau_{\text {im }}$ in the half-

space (see Figure $3(\mathrm{a})$ ) for different surface configurations. As expected, a free 
surface $\left(\mathrm{C}_{\mathrm{ijkl}}^{\text {Layer }}=\mathrm{C}_{\mathrm{ijkl}}^{\mathrm{I}}\right)$ has always an attractive effect on the dislocation whereas a rigid surface has a repulsive effect. However, $\tau_{\text {im }}$ exhibits a change of sign and a maximum when a stiff layer adjacent to the free surface is considered. This is explained by the opposite effects given by the stiff layer and the free surface on the dislocation image stress. For $\lambda$ much greater than 1 (Eq. 9), the stiff layer has a repulsive effect on the dislocation (Chen et al., 2019a). Therefore, $\tau_{\text {im }}$ is negative close to the stiff layer. However, it becomes positive when the dislocation is far away from the stiff layer due to the long range attractive effect of the free surface. When $\tau_{\text {im }}$ is positive, it increases to a maximum value and then decreases again, to become close to the value of the image stress corresponding to a free surface configuration (Figure 4). Thus, the effects of the free surface are felt at a longer distance compared to the effects of the stiff layer. These results show that the free surface effect becomes predominant at a long distance. This distance depends on both the thickness $h_{\text {Layer }}$ and the stiffness parameter $\lambda$ (Figure 4). It can be found that this distance increases with $h_{\text {Layer }}$ and $\lambda$. Figure 4 shows that the stiff layer's effect is predominant only when the dislocation is close to it. The influence of a stiff layer adjacent to a free surface on $\tau_{\text {im }}$ is totally different from the one of a rigid or free surface. For points close to the stiff layer, a similar behavior as a rigid surface is retrieved. In contrast, even for a large stiffness $\lambda=5$ or a large thickness $h_{\text {Layer }}=50 \mathrm{~nm}$, a similar behavior as a free surface is retrieved at a long distance from the stiff layer. 


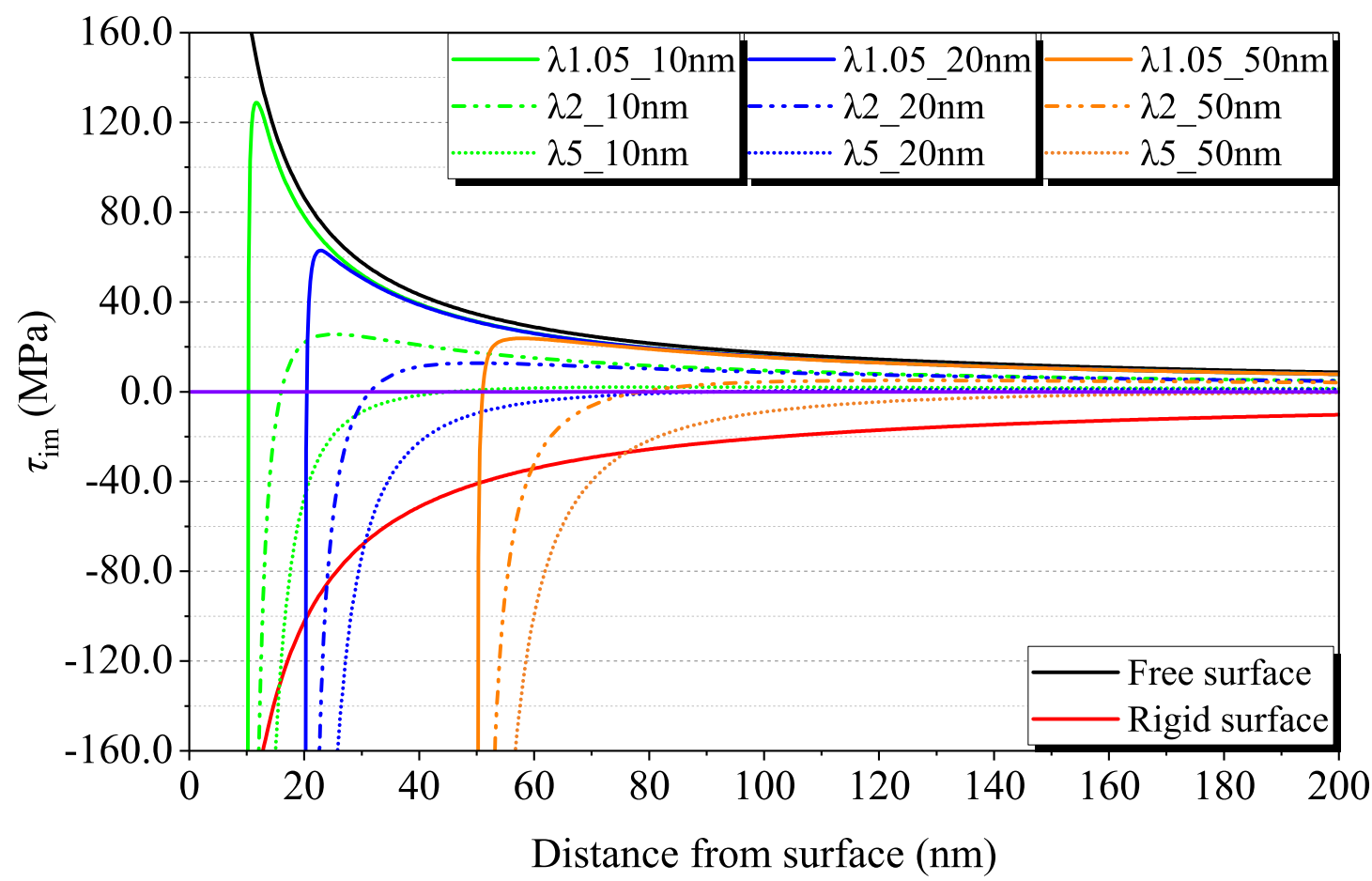

Figure 4: Variation of the image resolved shear stress $\tau_{\text {im }}$ in the half-space for different surface configurations. The stiff layer configuration is defined by the stiffness parameter $\lambda$ (see Eq. 9) and its thickness $h_{\text {Layer }}$ in nm (see Figure 3 (a)).

\subsubsection{Bi-crystal surface layer configuration: effects of two stiff layers adjacent to free surfaces and misorientation}

Figure 5 shows the variation of $\tau_{\text {im }}$ considering the layer stiffness effect on both free surfaces, together with the misorientation effect in the bi-crystal surface layer configuration shown in Figure 3 (b). The thicknesses of the two stiff layers are $h_{\text {Layer }}=10 \mathrm{~nm}$ and their stiffness parameter is $\lambda=2$ (see Eq. 9). The thicknesses of both crystals are $h=1990 \mathrm{~nm}$ so that the total thickness of the crystal on each side of the GB is $2 \mu \mathrm{m}$. Similarly to the previous observations, there is a sudden change of image stress close to the stiff layer due to the combined effects of the stiff 
layer and the free surface. Then, it is found out that the misorientation effect is only predominant in the area close to GB whereas the effect of the stiff layer is only predominant near the stiff layer (Figure 5). In most part of the crystal, $\tau_{\text {im }}$ is negative due to the top free surface whatever the misorientation. Furthermore, comparing the result obtained with just two free surfaces (marked as "Free surface_B Ori" in Figure 5 ), it is seen that the free surface effect at long distance is reduced in presence of stiff layers. As expected, the free surface effect is much weaker when $h_{\text {Layer }}$ or $\lambda$ gets larger.

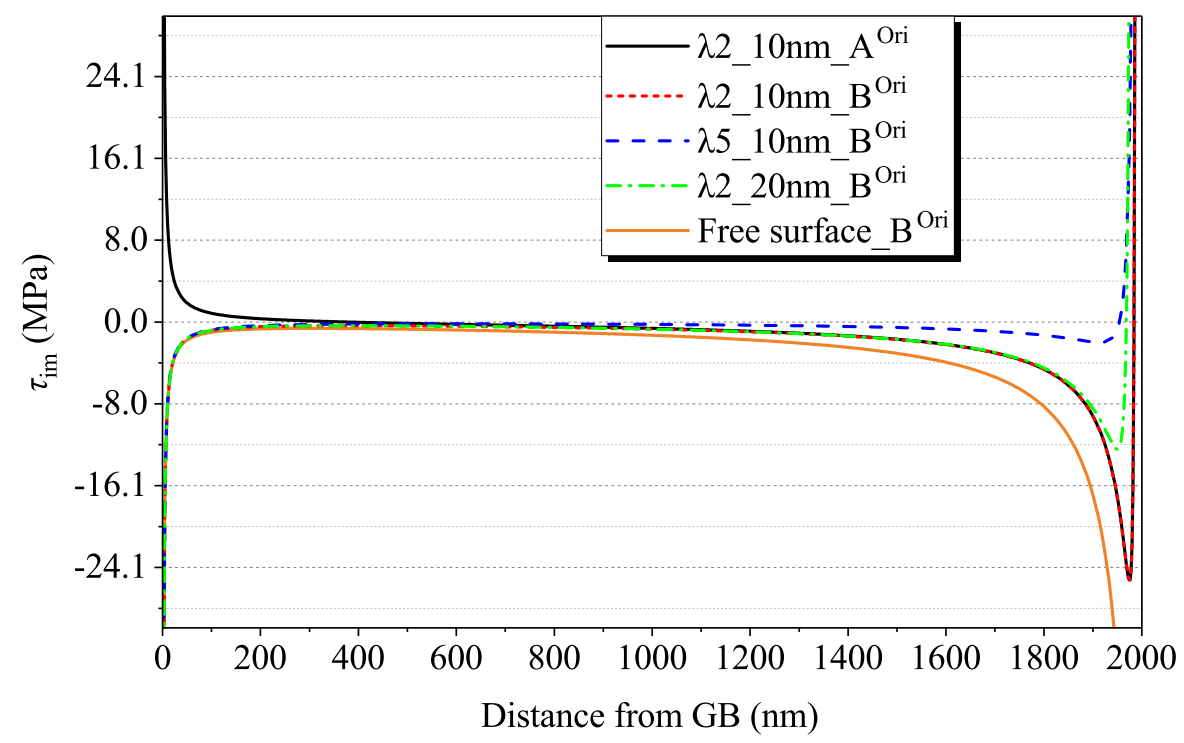

Figure 5: Variation of the image resolved shear stress $\tau_{\mathrm{im}}$ with the bi-crystal surface layer configuration considering stiff layer effects with attractive orientation $\mathrm{A}^{\text {Ori }}$ and repulsive orientation $\mathrm{B}^{\text {Ori }}$ of the bottom crystal. The stiff layer configuration is defined by the stiffness parameter $\lambda$ (see Eq. 9) and its thickness $h_{\text {Layer }}$ in nm (see Figure 3 (b)). As a comparison, the result for the configuration with two free surfaces is also presented and marked as "Free surface_B ${ }^{\text {Ori" ". }}$ 


\subsubsection{Bi-crystal free surface configuration: size effect on image stress}

The effects of free surfaces $\Lambda_{1}$ and $\Lambda_{2}$ on image stress are investigated by a 2layers configuration with different misorientations $\mathrm{A}^{\text {Ori }}, \mathrm{B}^{\text {Ori }}$ and different thicknesses $h=1 \mu \mathrm{m}$ and $h=2 \mu \mathrm{m}$ as shown in Figure 3 (c). Figure 6 shows that free surfaces have a much stronger attractive effect than the misorientation as the value of $\tau_{\text {im }}$ is quite similar close to the free surface whatever the GB misorientation.

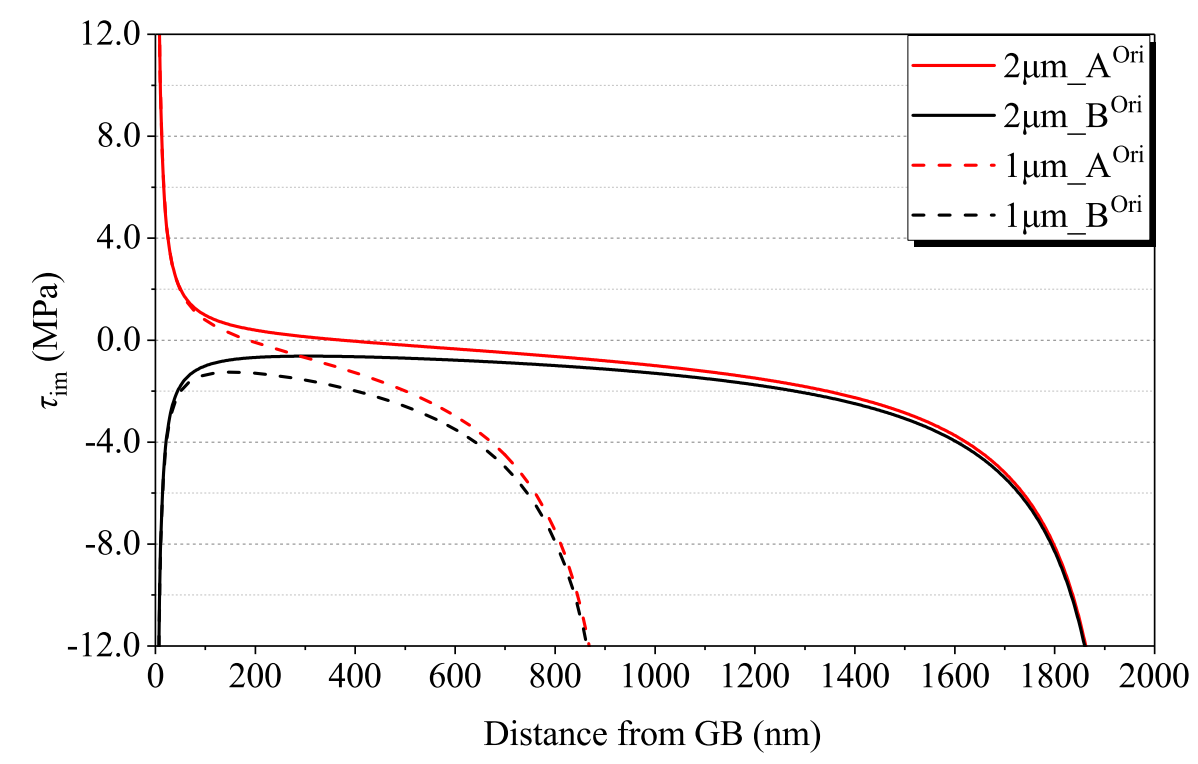

Figure 6: Variation of the image resolved shear stress $\tau_{\text {im }}$ with the distance from the GB for the bi-crystal free surface configuration with two free surfaces for different misorientations and crystal thicknesses $h$.

Furthermore, a clear size effect on the image stress is found after normalizing the distance from GB by $h$ as shown in Figure 7 for $A^{\text {Ori }}$ (Figure 7 (a)) and for $B^{\text {Ori }}$ (Figure 7 (b)). At a same relative distance from the GB, the image resolved shear stress magnitude is stronger in the smaller beam. This size effect arises because the image stress depends on a characteristic material internal length which is the 
Burgers vector magnitude $|\boldsymbol{b}| .|\boldsymbol{b}|$ is indeed an intrinsic material length that does not change with $h$. Meanwhile, in the case of the attractive misorientation $\mathrm{A}^{\text {Ori }}$, there is an equilibrium point where the image resolved shear stress on the dislocation is zero. This position is located at nearly the same relative distance from GB for different thicknesses of material as shown in Figure 7 (a). In contrast, the case of repulsive misorientation $\mathrm{B}^{\text {Ori }}$ does not lead to an equilibrium point, see Figure 7 (b). 

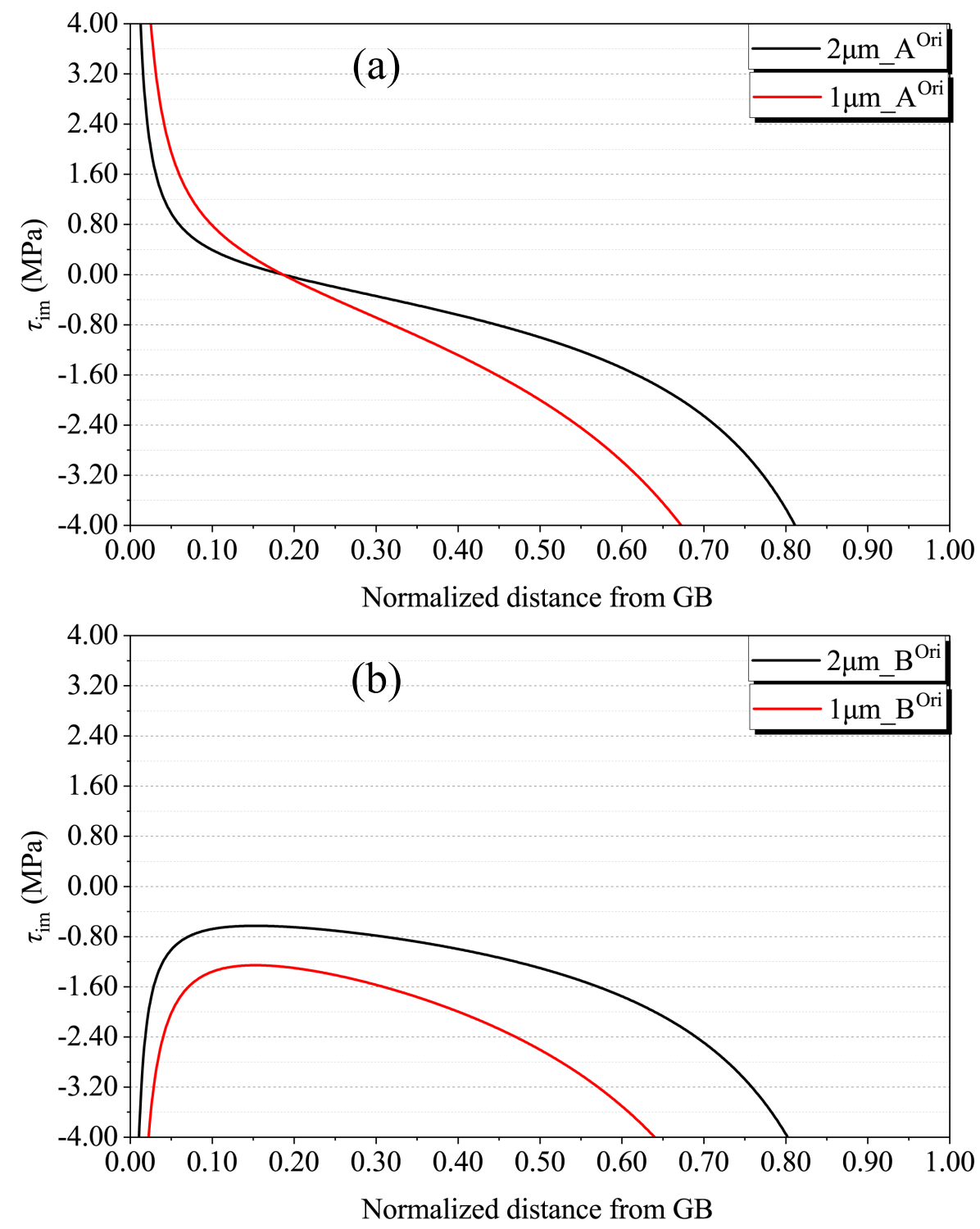

Figure 7: Variation of the image resolved shear stress $\tau_{\text {im }}$ with the distance from GB normalized by $h$ for the bi-crystal free surface configuration with two free surfaces (see Figure 3 (c)): (a) with attractive misorientation $\mathrm{A}^{\text {Ori }}$ and (b) with repulsive misorientation $\mathrm{B}^{\text {Ori }}$. The $1 \mu \mathrm{m}$ and $2 \mu \mathrm{m}$ indicate the crystal thickness $h$. 


\subsubsection{Analysis of the specific role of top and bottom free surfaces and misorientation}

In order to specify the effect of each free surface and misorientation within the configuration of Figure 3 (c) (Bi-crystal free surface configuration) with $h=2 \mu \mathrm{m}$, $\tau_{\text {im }}$ is computed either with only one top free surface or with only one bottom free surface as shown in Figure 8. It is shown that close to GB, $\tau_{\text {im }}$ is mainly controlled by the misorientation as it is always positive for the misorientation $\mathrm{A}^{\text {Ori }}$ (see Figure 8 (a)) and always negative for the misorientation $B^{\text {Ori }}$ (see Figure 8 (b)) whatever the free surface configuration. The effects of both free surfaces and misorientation can be regarded as the non-linear combination of both effects. As the two free surfaces have an opposite effect on the dislocation, when the dislocation is located roughly at an equal distance from both free surfaces, their contributions become negligible. For the configuration with both free surfaces ("Two free surfaces"), when the dislocation is located close to GB i.e., for a distance from GB smaller than $0.15 \mu \mathrm{m}$, the value of $\tau_{\text {im }}$ is similar as in the case of a bi-crystal configuration without free surface for both misorientations $\mathrm{A}^{\text {Ori }}$ and $\mathrm{B}^{\text {Ori }}$ ("Infinite bi-crystal"). However, if only one free surface is considered, it has an obvious influence on the image stress even close to GB. Figure 8 (a) shows that the positive $\tau_{\text {im }}$ due to attractive misorientation $\mathrm{A}^{\text {Ori }}$ is enhanced by considering only the bottom free surface ("Bottom free surface_A Ori"), which also has an attractive effect on the dislocation. Meanwhile, by considering only the top free surface, this positive $\tau_{\mathrm{im}}$ is weakened close to GB ("Top free surface_A Ori") and an equilibrium position is found $\left(\tau_{\mathrm{im}}=0\right)$. In particular, an interesting result is obtained by combining a repulsive misorientation $\mathrm{B}^{\text {Ori }}$ with just a bottom free surface ("Bottom free surface_B Ori") as shown in Figure 8 (b). It is shown that $\tau_{\text {im }}$ is negative close to $\mathrm{GB}$ due to repulsive misorientation $\mathrm{B}^{\text {Ori }}$, then becomes positive away from GB (at a distance of about $0.1 \mu \mathrm{m}$ ) due to the attractive effect of the 
bottom free surface, whereas the distances between the dislocation and both GB and bottom free surface increase at the same time. As discussed for Figure 6, the effects of a free surface are felt at a longer distance compared to the misorientation effects. However, Figure 8 shows that the effect of misorientation is predominant when the dislocation is close to GB, but only within a short distance. Thus, at a long distance, the free surface effect is predominant. This result is quite similar to the case of a stiff layer close to the free surface. It can be found that the free surface displays the highest distance over which the image stress is significant. Hence, the effect of a free surface is always predominant when the dislocation is far away from stiff layers and GB. Furthermore, for the case of a repulsive misorientation $B^{\text {Ori }}$ with only a top free surface ("Top free surface_B ${ }^{\text {Ori”", }}$, see Figure $8(\mathrm{~b})$ ), the magnitude of $\tau_{\text {im }}$ decreases quickly when the dislocation gets away from GB at a short distance from it. In particular, it should be pointed out that the analyzed dislocation is always in the crystal I, therefore the distance between the dislocation and the top free surface is reduced when dislocation gets away from GB, but it increases for the distance between the dislocation and the bottom free surface. Hence, the effects of the top free surface and the bottom free surface get stronger and weaker, respectively, when the dislocation gets away from GB in crystal I (see Figure 8). 

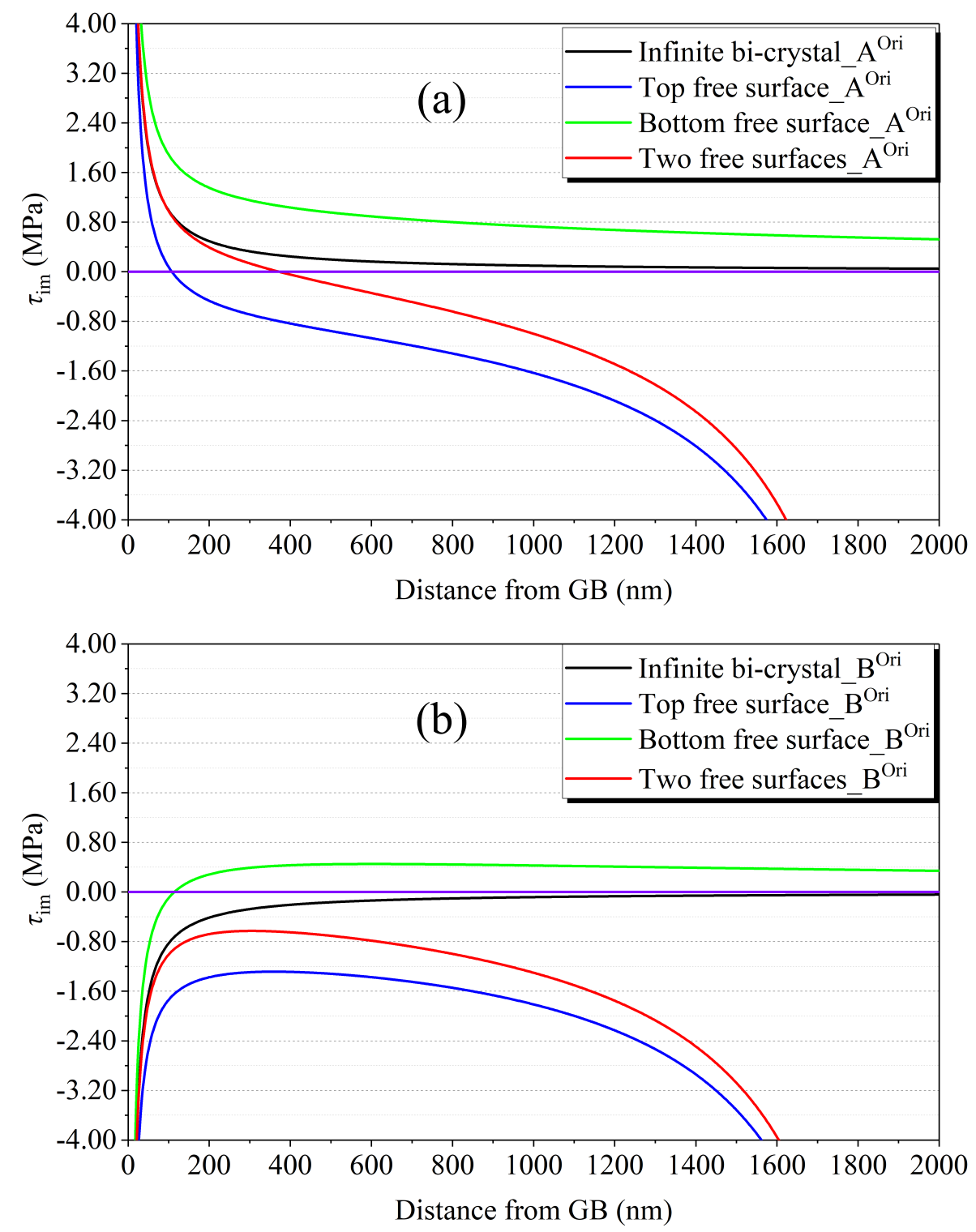

Figure 8: Variation of the image resolved shear stress $\tau_{\mathrm{im}}$ for the bi-crystal free surface configuration considering free surface effects with different misorientations (see Figure 3 (c)): (a) attractive orientation $\mathrm{A}^{\text {Ori }}$ and (b) repulsive orientation $\mathrm{B}^{\text {Ori }}$ of the bottom crystal. 


\subsubsection{Infinite tri-crystal configuration: grain size effect on the image stress}

In addition to the analysis of the finite size effect for a bi-crystal bounded by two parallel free surfaces (see Figure 3 (c)), the grain size effect of a grain bounded by two parallel GBs in an infinite tri-crystal configuration is also studied (see Figure 3 (d)). Figure 9 shows the variation of the image resolved shear stress $\tau_{\text {im }}$ on the dislocation in the middle crystal for different thicknesses and different misorientations with top and bottom crystals. It should be pointed out that the sign convention of $\tau_{\mathrm{im}}$ is given by a glide direction $\boldsymbol{v}$ pointing towards the second GB $\Gamma_{2}$ (see Figure $3(\mathrm{~d})$ ). Hence, when GBs have the same misorientations, there is an equilibrium position of the dislocation $\left(\tau_{\mathrm{im}}=0\right)$ as shown in Figures $9(\mathrm{a}-\mathrm{b})$. The two opposite image stresses arising from each GB balance each other at the middle of the grain. Furthermore, if both GBs have opposite misorientation effects, image stresses in the same direction add up as shown in Figures 9 (c-d). As discussed previously, the effective distance of misorientation effects is short, so the magnitude of the image stress decreases when the distance from the dislocation to the closest GB increases.

Similarly to the case of two free surfaces as shown in Figure 7, a size effect is also found out within the present configuration when two different thicknesses of the middle crystal are considered (Figure 9). The magnitude of the image resolved shear stress $\tau_{\text {im }}$ is indeed always larger at the same relative distance from GB within a thinner crystal, except around the equilibrium position. This size effect is present whatever the misorientation configurations of both GBs. Again, this grain size effect arises because the image stress scales with the intrinsic material length $|\boldsymbol{b}|$. 

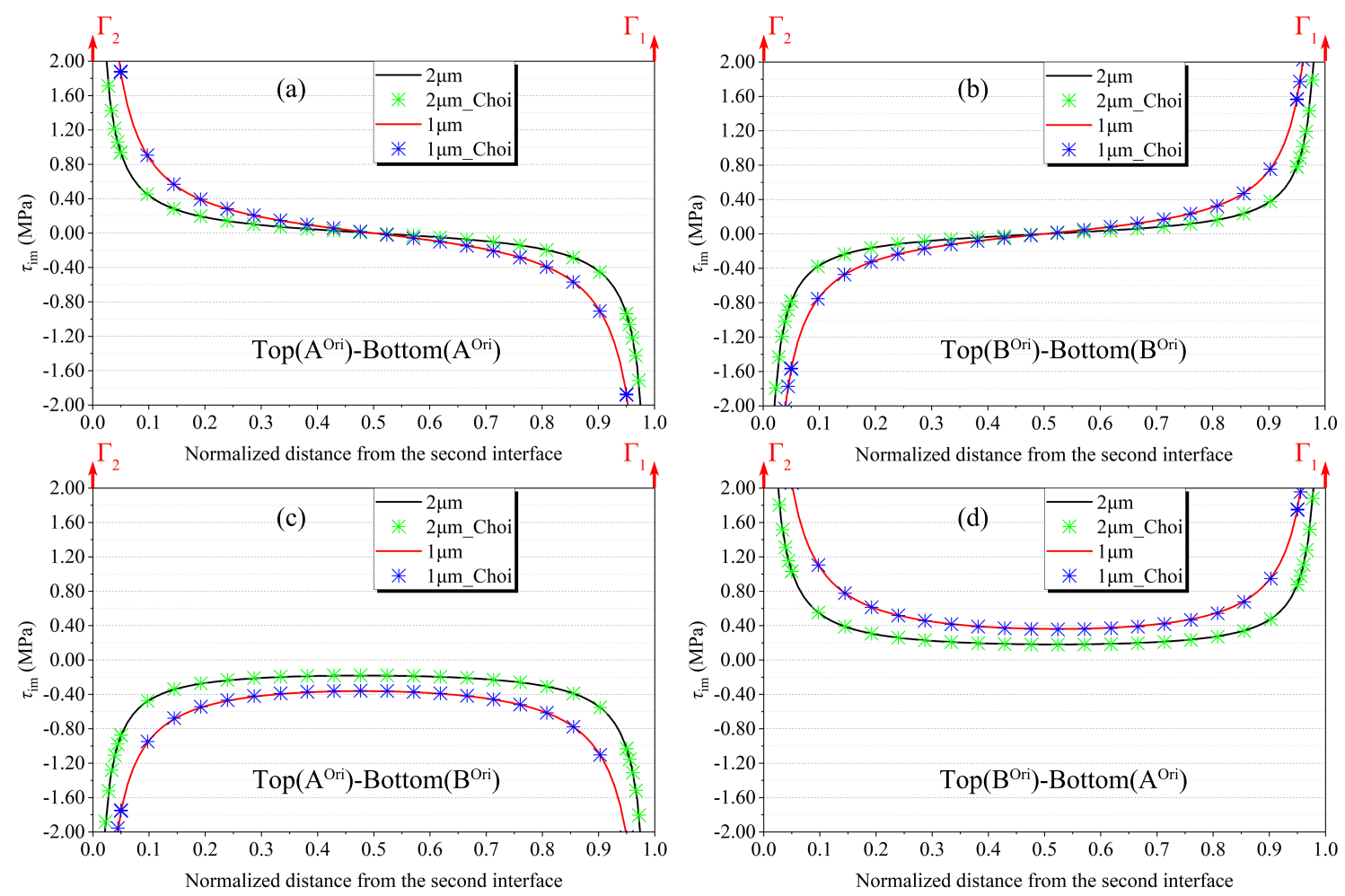

Figure 9: Variation of the image resolved shear stress $\tau_{\text {im }}$ with the distance from the second GB $\Gamma_{2}$ to the first GB $\Gamma_{1}$ normalized by the thickness of the middle crystal $h$ (see Figure 3 (d)). The crystallographic orientations of top and bottom crystals in the infinite tri-crystal configuration are marked on each result. The $1 \mu \mathrm{m}$ and $2 \mu \mathrm{m}$ indicate the thickness of the middle crystal $h$. The current results (solid lines), obtained by the image decomposition method of Wang et al. (2007), are compared with those, marked by symbols, obtained by the alternating technique of Choi and Earmme (2002a).

\subsection{Analysis of double-ended discrete dislocation pile-ups with the Bi-crystal surface layer configuration}

As indicated in the introduction, a double-ended dislocation pile-up has been recently observed from post-mortem slip step height measurements in a compressed bi-crystalline $\alpha$-Brass micro-pillar (Chen et al., 2020). In that case, dislocations 
are pilling-up both at GB and at an external surface. Therefore, we assume the presence of a layer stiffer than the bulk material in order to stabilize the dislocation pile-up. Hence, the second application of this study is about dislocation distribution in double-ended dislocation pile-ups with different simulation configurations. For a general case, the equilibrium positions of the dislocations in the pile-up can be found out by minimizing the component of the Peach-Koehler $(\mathrm{P}-\mathrm{K})$ force along the glide direction on each dislocation to a material's critical force $F_{c}$ as follows (Wagoner, 1981; Chen et al., 2020):

$$
F^{(\gamma)}=\left\{\left(\boldsymbol{\sigma}_{\text {int }}\left(X_{1}(\gamma), X_{2}(\gamma)\right)+\boldsymbol{\sigma}_{\text {ext }}\right) \cdot \boldsymbol{b} \times \boldsymbol{t}\right\} \cdot \boldsymbol{v} \stackrel{\text { Minimize }}{=} F_{c}
$$

where $\left(X_{1}(\gamma), X_{2}(\gamma)\right)$ denotes the position of the $\gamma^{\text {th }}$ dislocation. $\boldsymbol{\sigma}_{\text {int }}$ is the stress tensor coming from all the other dislocations and all heterogeneities, involving interfaces and surfaces. $\boldsymbol{\sigma}_{\text {ext }}, \boldsymbol{b}, \boldsymbol{t}$ and $\boldsymbol{v}$ are the applied stress tensor, the Burgers vector, the dislocation line vector and the glide direction, respectively. Similarly to the image resolved shear stress defined in Eq. 11, the critical shear stress of the material can be defined as $\tau_{c}=F_{c} /|\boldsymbol{b}|$.

\subsubsection{Frank-Read dislocation sources}

There are many different dislocation sources, such as Frank-Read sources, particles and inclusions, GBs, surfaces or cracks (Li, 1981). In the present paper, the Frank-Read source is chosen as the dislocation source for dislocation pile-ups, which is the most common mechanism. The Frank-Read source produces dislocation loops from two pinned points $\mathrm{A}$ and $\mathrm{B}$ under shear stress in a finite elastic medium as shown in Figure 10 (a) by the yellow circle line. However, a dislocation loop may be broken into two curved dislocation segments when it encounters some external boundaries (see the two orange curves in Figure 10 (a)). These dislocation segments 
belong to the same slip plane but have opposite signs. It should be pointed out that these boundaries are not the GB or the surfaces considered in the micromechanical calculations. These external boundaries are described in Figure 10 (b). Furthermore, in order to apply two-dimensional calculations, the dislocation segments are regarded as parallel infinite straight lines as shown in Figure 10 (b) with black lines and the dislocation segment $\mathrm{AB}$ is regarded as a pinned dislocation as indicated in Figure 10 (b).
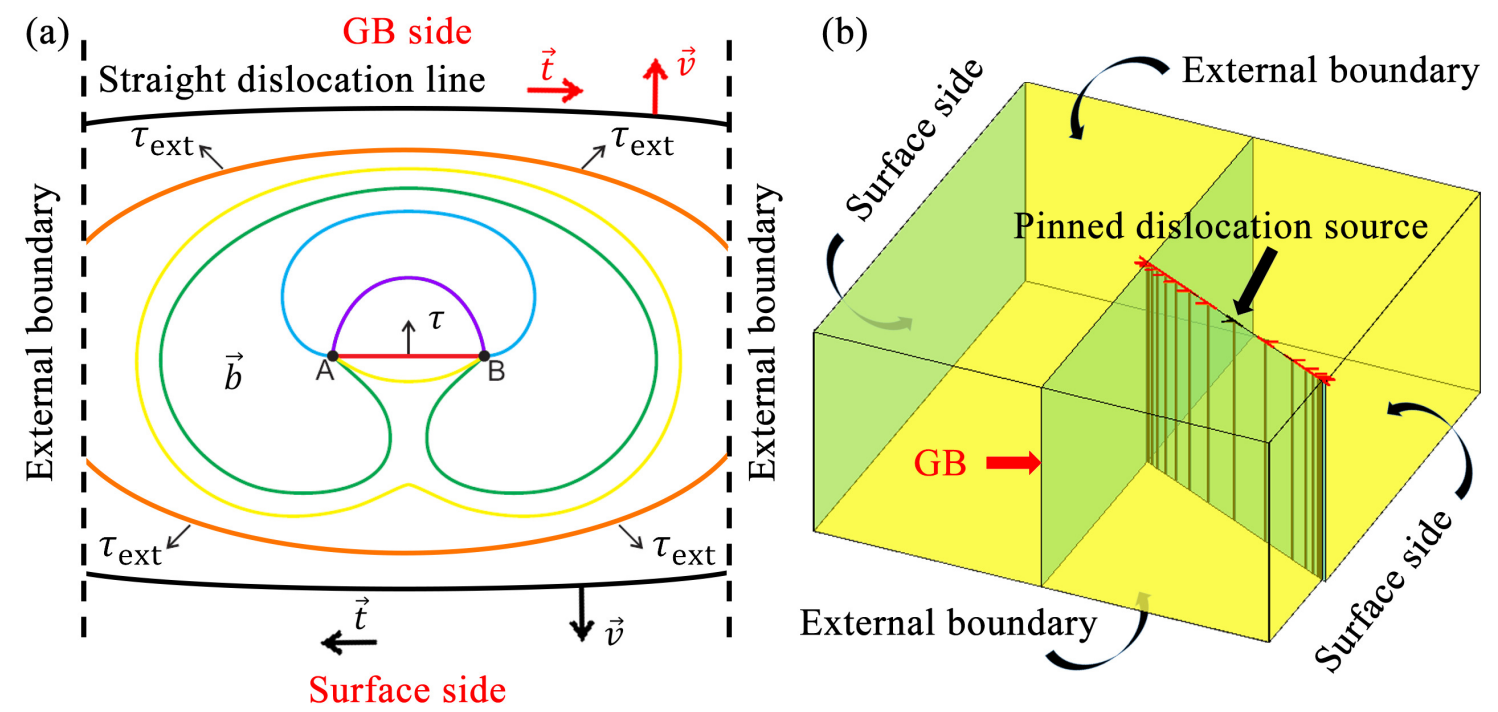

Figure 10: Schematic figure of (a) a Frank-Read dislocation source used for (b) two-dimensional configuration in a bi-crystal model with external boundaries.

\subsubsection{Configurations of double discrete dislocation pile-ups with Frank-Read disloca- tion source}

In order to create a stable double dislocation pile-up close to GB and surface, repulsive forces on dislocations (coming from a repulsive misorientation and/or a stiff layer) must be provided. Hence the surface can be modeled as a rigid surface or as a stiff layer adjacent to the free surface. In the case of a free surface, no dislocation 
pile-up can be built-up as the attractive image stress make all the dislocations get out of the material and create a step on the surface. Even though the developed model is not dynamic, it can still be considered that dislocations are produced from a Frank-Read source during the loading process as described in subsection 3.2.1. Thus, dislocations of opposite signs pile up on both sides of the dislocation source and it is supposed that there is one pinned dislocation at the dislocation source position. The algorithm during the loading process is presented in Figure 11 (a). A pair of dislocations with opposite signs are introduced on both sides of the pinned dislocation until the absolute value of the resolved shear stress $\left|\tau_{s}\right|$ on the pinned dislocation is smaller than $\tau_{c}$, once all the dislocations have reached their equilibrium position as described in Figure 12 (a). However, in the case where no equilibrium can be reached after having introduced the last two opposite dislocations, these ones are not considered and the pinned dislocation is moved towards the GB. Then, equilibrium dislocation positions including the pinned dislocation are calculated again. This last process can be understood as the case where the applied stress cannot produced an entire dislocation loop at the end, but the pinned dislocation is bowed out as shown in Figure 10 (a) (see e.g. the purple curve or the blue curve). 

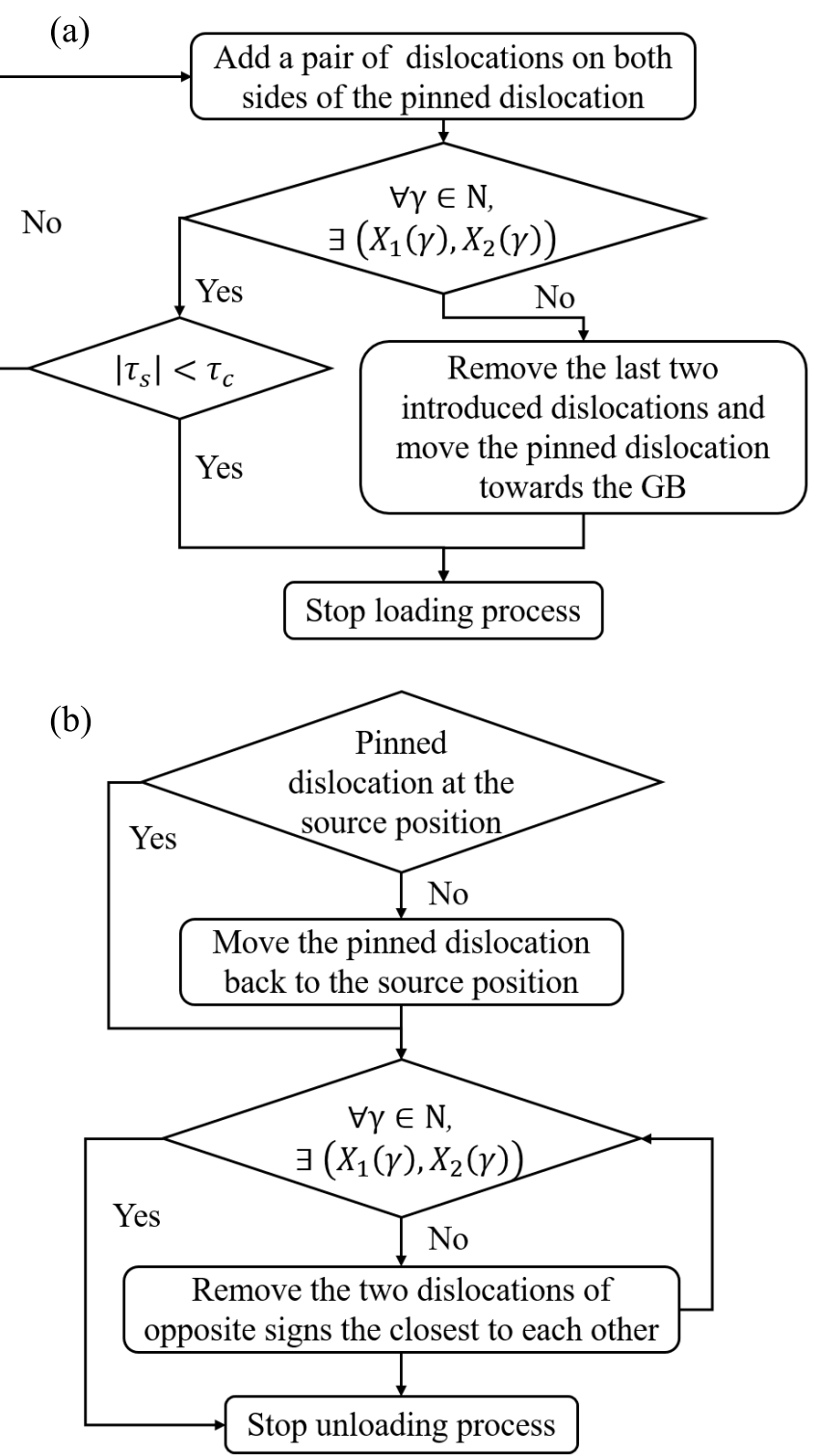

Figure 11: Simulation algorithms to equilibriate a double discrete dislocation pile-up during (a) loading, (b) unloading. $N$ indicates the total number of dislocations in the pile-up and $\left(X_{1}(\gamma), X_{2}(\gamma)\right)$ indicates the equilibrium position of the $\gamma^{\text {th }}$ dislocation.

At unloading, the applied stress is removed and all the dislocations should the- 
oretically move back due to the internal stress tensor $\boldsymbol{\sigma}_{\text {int }}$, which involves the backstresses from dislocations, image stresses and also the attractive stresses between dislocations with opposite signs. In particular, the annihilation of dislocations with opposite signs should occur when their separation distance is below a critical value. However, it is reminded that the developed model is not dynamic and a static equilibrium position calculation is performed by solving a system of nonlinear equations using the "fsolve" function in MATLAB. Hence, within our model, two opposite dislocations cannot be too close to each other due to the increasing attractive stress between them. As a consequence, the annihilation of opposite dislocations at unloading is carried out by removing a pair of opposite dislocations when no equilibrium is reached. The algorithm for the unloading process is presented in Figure 11 (b). It should be pointed out that in the case where the pinned dislocation is bowed out at the end of loading, then it is firstly moved back to the source position at the beginning of the unloading process. 

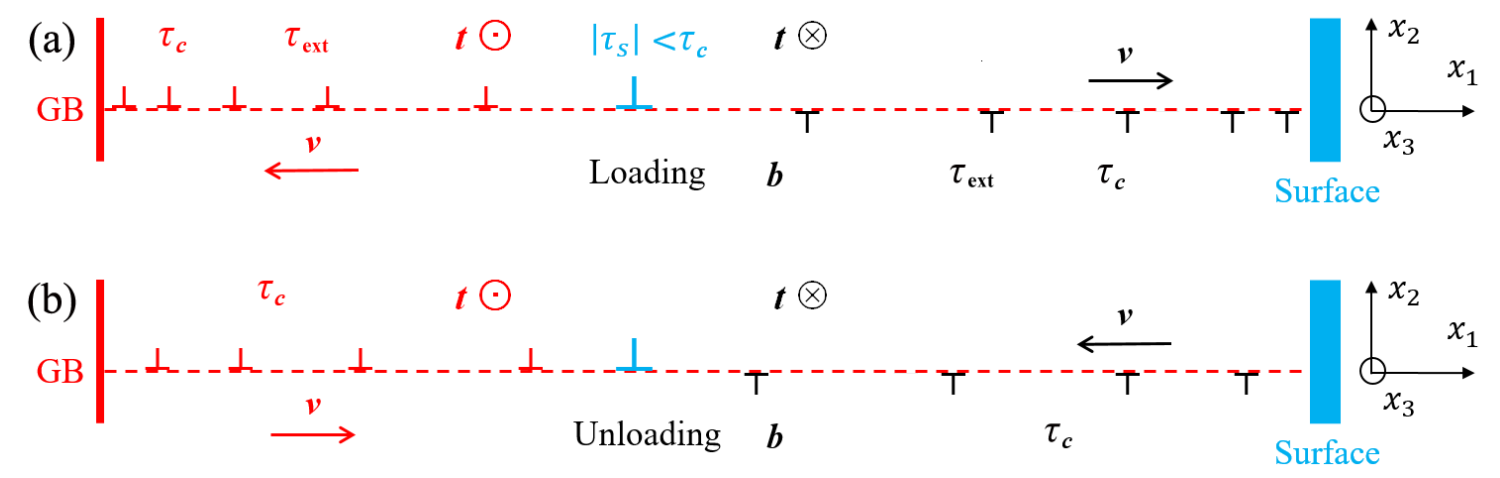

Figure 12: Schematic figure of a double discrete dislocation pile-up configuration in a bi-crystal surface layer configuration assuming a Frank-Read dislocation source. (a) At loading, the dislocation source produces dislocations both towards the GB and the surface until all dislocations are finally equilibrated. (b) At unloading, all dislocations move back towards the dislocation source due to internal stresses (backstresses). In order to consider a double dislocation pile-up, the surface condition can be set to be a rigid surface or a stiff layer adjacent to the free surface, but not a free surface. $\tau_{\text {ext }}$ indicates the applied resolved shear stress and the rest of the notations are the same as described in the previous sections.

In order to create a stable double dislocation pile-up with the proposed method, only repulsive misorientation $\mathrm{B}^{\text {Ori }}$ is chosen and the surface condition is defined as a stiff layer adjacent to free surface or a rigid surface, which both can provide a repulsive force on dislocations. In the case of bi-crystal surface layer configuration, $h=7990 \mathrm{~nm}, h_{\text {Layer }}=10 \mathrm{~nm}$ and $\lambda=2$ (see Eq. 9) as shown in Figure 13(a). For the bi-crystal rigid surface configuration, $h=8000 \mathrm{~nm}$ and surfaces $\Lambda_{1}$ and $\Lambda_{2}$ are displacement free as shown in Figure 13 (b). The source position is always located in the center of crystal I. During loading, the applied stress is $\sigma_{11}=30 \mathrm{MPa}$. Then, the corresponding applied resolved shear stress is $\tau_{\text {ext }}=15 \mathrm{MPa}$ for the slip system defined with $\alpha=45^{\circ}$ (see Figure 13). The other simulation configurations are the same as described in subsection 3.1.2. However, two glide directions $\boldsymbol{v}$ must be 
considered as described in Figure 12. Furthermore, for the forthcoming results, the distance from GB is the distance along the glide direction.

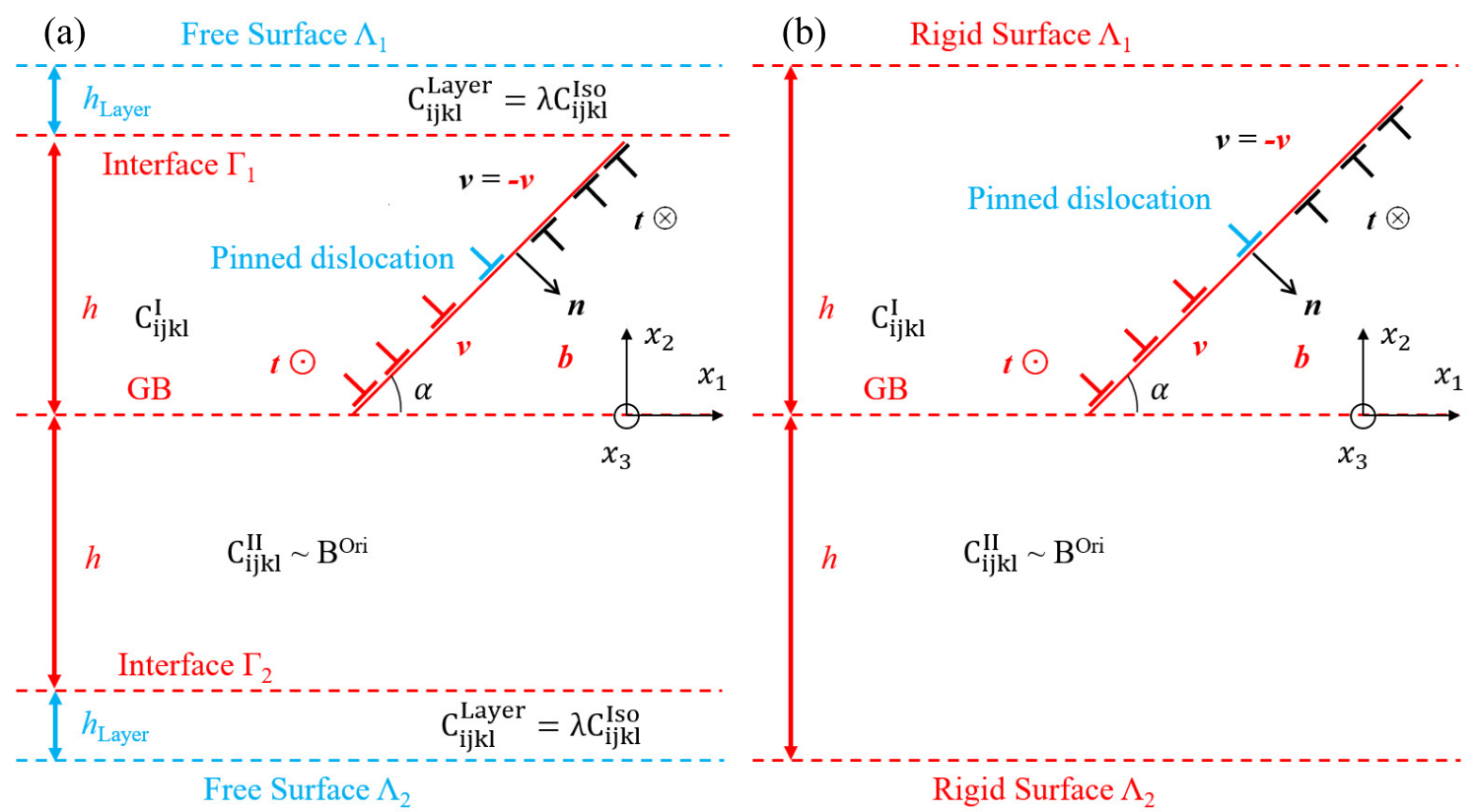

Figure 13: $\alpha$-inclined double discrete dislocation pile-up in a slip plane of unit normal $\boldsymbol{n}$ for $N$ equilibrated edge dislocations with Burgers vector $\boldsymbol{b}$ and line vector $\boldsymbol{t}$ assuming: (a) stiff layers adjacent to free surfaces, (b) rigid surfaces

\subsubsection{Dislocation distributions in double discrete dislocation pile-ups}

Figure 14 (a) shows the dislocation distribution with the bi-crystal surface layer configuration. At loading, it is found that a double dislocation pile-up is always formed ahead of the GB and the stiff layer for different values of critical stress. Besides, it is also observed that the dislocation density next to the stiff layer is always higher than the one close to the GB. Indeed, the long range attractive image stress due to the free surface attracts dislocations towards the surface and increases the dislocation density (see the profile of the image resolved shear stress in Figure 5). 
In contrast, the repulsive GB displays only a repulsive effect on dislocations, and so reduces the dislocation density close to the GB. Regarding the critical stress effect, the dislocation density decreases when the critical stress increases. The physical reason is that a high critical stress always hinders dislocation motion and thus weakens the applied stress. Then, at unloading, the number of dislocations decreases when the critical stress decreases due to easy unpilling-up of dislocations. For example, with $\tau_{c}=1 \mathrm{MPa}$, all the dislocations are removed after unloading as shown in Figure 14 (a). However, there are more dislocations remaining stored in the pile-up with a higher $\tau_{c}$ (see Figure $14(\mathrm{a})$ ). Since the critical stress always opposes to dislocation motion, it hinders reverse dislocation motion during unloading and so reduces dislocation annihilation during the unpilling-up mechanism.

For comparison, Figure 14 (b) shows the results with bi-crystal rigid surface configuration. With this configuration, there are also double dislocation pile-ups both after loading and unloading. The effects of loading/unloading and of the critical stress are qualitatively the same as with the bi-crystal surface layer configuration. However, the dislocation density in the pile-up is lower with rigid surfaces compared to stiff layers for the same simulation conditions. In the case of a stiff layer, a dislocation pile-up can be easily built as the attractive image stress due to the free surface attracts dislocations towards the surface and close to the stiff layer, the repulsive force stabilizes the dislocations. In contrast, a bi-crystal rigid surface configuration displays only a repulsive effect against the dislocations canceling the long range free surface attractive effect. Therefore, the dislocation density in the pile-up is smaller. Furthermore, the dislocation density is now lower close to the rigid surface and higher close to the GB. The repulsive effect of a rigid surface is indeed much stronger than the one of a repulsive GB (Chen et al., 2019a).

Besides, it is interesting to show that with a critical stress $\tau_{c}=8 \mathrm{MPa}$ and 
an applied resolved shear stress $\tau_{\mathrm{ext}}=15 \mathrm{MPa}$, the equilibrium positions of all dislocations do not change at unloading whatever the surface conditions as shown in Figure 14. The effective shear stress on each dislocation at unloading is $\tau=$ $\left|\tau_{\text {ext }}\right|-\tau_{c}=7 \mathrm{MPa}$ which is smaller than the critical stress $\tau_{c}$. Therefore, the driving force at unloading is smaller than the critical stress, and so the equilibrium positions of dislocations do not change. 

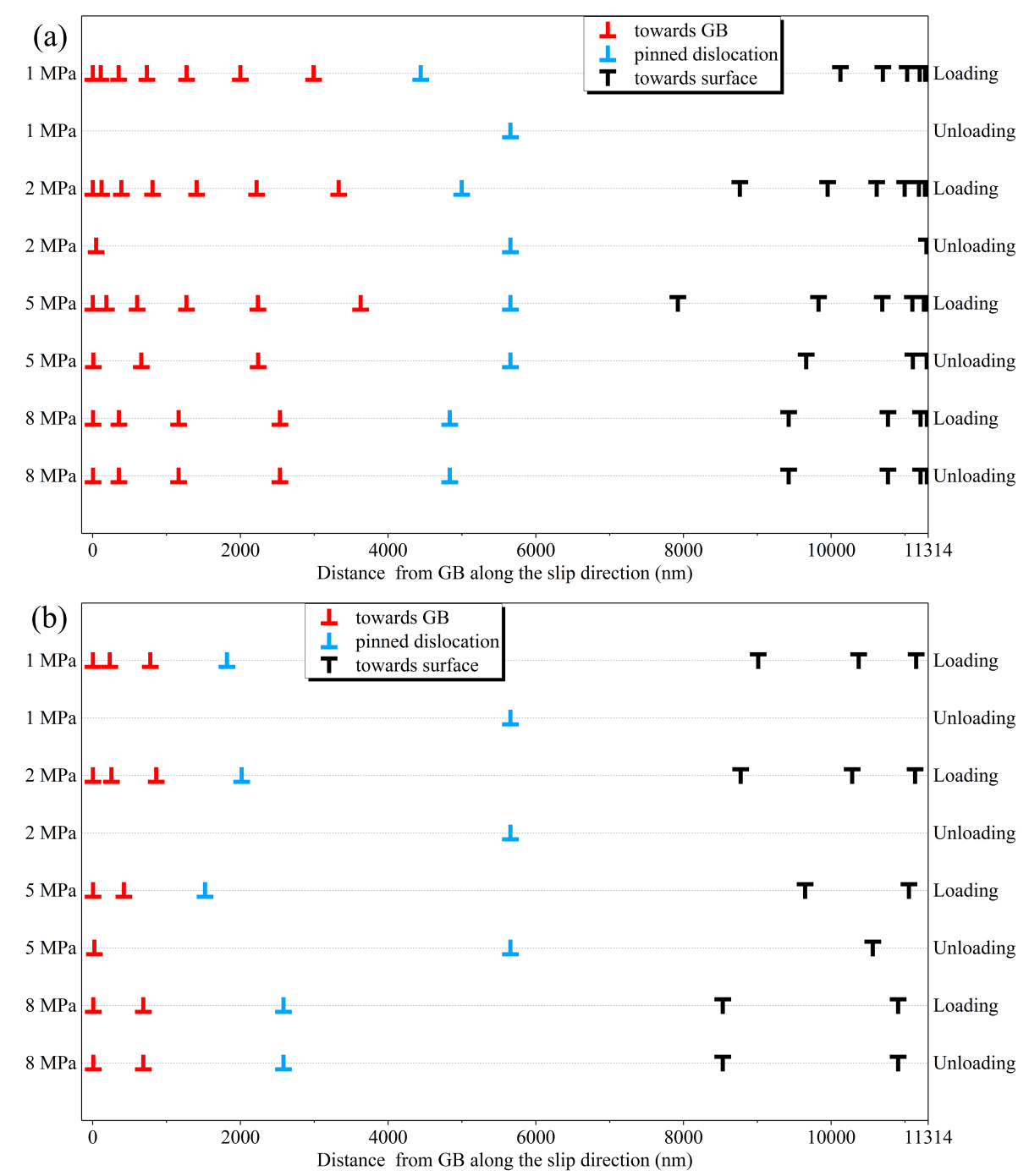

Figure 14: Dislocation equilibrium positions in double-ended dislocation pile-ups with a FrankRead dislocation source for different critical stresses $\tau_{c}$ during loading and unloading processes: (a) bi-crystal surface layer configuration and (b) bi-crystal rigid surface configuration. The left Y axis indicates the critical stress value $\tau_{c}$ and the right $\mathrm{Y}$ axis indicates the loading state. 


\section{Conclusions and perspectives}

The present paper is an application of the image decomposition method developed by Wu and Wang (2007) for two-dimensional anisotropic elasticity to compute elastic fields of single dislocations and discrete dislocation pile-ups in different bi-crystal configurations in the presence of surfaces. A new bi-crystal surface layer configuration is proposed, where a stiff layer of finite thickness adjacent to the free surface is introduced. This stiff layer is characterized by two quantities, which are its thickness and its stiffness given by a scalar parameter $\lambda$.

Based on the analysis of image resolved shear stress on dislocations in $\mathrm{Ni}$, the bi-crystal surface layer configuration with a stiff layer shows significant differences compared to a bi-crystal rigid surface configuration or free surface configuration. In particular, it is found that the distances over which free surface, grain misorientation and stiff layer have an effect on a dislocation can be classified as follows: free/rigid surface $>$ GB misorientation $>$ stiff layer. However, the effects of each heterogeneity are always predominant at a short distance around them. Besides, a finite size effect on the dislocation image stress is reported when considering two parallel free surfaces. Meanwhile, a grain size effect is also found when considering two planar GBs in an infinite tri-crystal configuration. Both size effects are due to the scaling of the image stress with the Burgers vector magnitude $|\boldsymbol{b}|$.

In addition, a double discrete dislocation pile-up model is proposed based on the Frank-Read dislocation source mechanism. During loading, double dislocation pileups are built up depending on GB misorientation, surface layer stiffness and critical stress for dislocation slip. Then, at unloading, the annihilation of dislocations with opposite signs are described during the unpilling-up mechanism. Furthermore, the

dislocation density in the pile-up is higher when considering the bi-crystal surface 
layer configuration compared to the bi-crystal rigid surface configuration both after loading and unloading. However, the dislocation density in the pile-up is higher close to the stiff layer than close to the GB, while the reverse effect is observed with the bi-crystal rigid surface configuration. As expected, it is also found that with a higher critical stress for the same considered material $(\mathrm{Ni})$, there are less produced dislocations during loading. However, at unloading, a higher critical stress reduces or even impedes dislocation annihilation. Thus, the final dislocation density on both sides, as the one that can be observed in post-mortem experiments, may be higher than the one resulting from a lower critical stress. In the present study, the critical stress of the crystalline material is regarded as a homogeneous value, although it may change according to the distribution of internal defects in the material. Thus, a value of critical stress associated with the local dislocation density could be considered in a future study.

Furthermore, the developed numerical code can be used for two-dimensional DDD calculations and the new bi-crystal surface layer configuration can be applied to study other issues such as the improvement of fatigue life by hard surface coatings (Dodaran et al., 2020) or the effect of passivated layers on the mechanical behavior of thin films (Nicola et al., 2006).

\section{Supplementary materials}

The developed MATLAB code for the image resolved shear stress calculation on one single dislocation in the bi-crystal with different surface conditions (as shown in the figure 3(b) of this paper) and with GB seen as an interphase with a given stiffness (Chen et al., 2019a), is provided at https://github.com/AniPlas/AniSurfDis. This code is based on the image decomposition method originally developed by Wang et al. (2007). The provided code can be easily adapted to simpler cases (planar GB 
without thickness or surface without stiff layer) but always with two outside free surfaces (surfaces $\Lambda_{1}$ and $\Lambda_{2}$ in the figure 3 (b) of this paper). Besides, the provided code can be used with multi-CPUs acceleration with MATLAB Parallel Computing Toolbox and with C-MEX acceleration (using the MATLAB Coder Toolbox within MATLAB 2018a or more recent versions).

\section{Funding}

The authors are grateful to the French Ministry of Higher Education and Scientific Research and the French-German University (UFA-DFH) for financial supports. The authors are also grateful to Experimental Methodology in Materials Science (MWW) in Saarland University and the French State (ANR) through the program Investment in the future (LabEx "DAMAS" referenced as ANR-11-LABX-0008-01) for additional financial supports. 
Anderson, P.M., Hirth, J.P., Lothe, J., 1968. Theory of Dislocations. Cambridge University Press.

Armstrong, R.W., 1970. The influence of polycrystal grain size on several mechanical properties of materials. Metallurgical and Materials Transactions B 1, 1169-1174.

Armstrong, R.W., 2014. 60 years of Hall-Petch: Past to present nano-scale connections. Materials Transactions 55, 2-12.

Belov, A.Y., 1992. Dislocations emerging at planar boundaries, in: Indenbom, V.L., Lothe, J. (Eds.), Modern Problems in Condensed Matter Sciences, Elastic Strain Fields and Dislocations Mobility. Pergamon. volume 31, pp. 432-438.

Berbenni, S., Taupin, V., Lebensohn, R.A., 2020. A fast fourier transform-based mesoscale field dislocation mechanics study of grain size effects and reversible plasticity in polycrystals. Journal of the Mechanics and Physics of Solids 135, 103808.

Beucia, B., Queyreau, S., Kahloun, C., Chaubet, D., Franciosi, P., Bacroix, B., 2020. Plastic strain-induced grain boundary migration (SIBM) in pure aluminum: SEM in-situ and AFM examinations. International Journal of Plasticity 134, 102776.

Borodin, E.N., Mayer, A.E., Gutkin, M.Y., 2017. Coupled model for grain rotation, dislocation plasticity and grain boundary sliding in fine-grained solids. International Journal of Plasticity 96, 227-241.

Borovikov, V., Mendelev, M.I., King, A.H., 2018. Effects of Ag and Zr solutes on dislocation emission from $\sigma 11(332)[110]$ symmetric tilt grain boundaries in $\mathrm{Cu}$ : Bigger is not always better. International Journal of Plasticity 109, $79-87$. 
Chen, X., 2020. Experimental and theoretical studies of incompatibility and dislocation pile-up stresses at grain boundaries accounting for elastic and plastic anisotropies. Ph.D. thesis. Université de Lorraine, Metz, France and Universität des Saarlandes, Saarbrücken, Germany.

Chen, X., Richeton, T., Motz, C., Berbenni, S., 2019a. Elastic fields due to dislocations in anisotropic bi- and tri-materials: Applications to discrete dislocation pile-ups at grain boundaries. International Journal of Solids and Structures 164, $141-156$.

Chen, X., Richeton, T., Motz, C., Berbenni, S., 2020. Atomic force microscopy study of discrete dislocation pile-ups at grain boundaries in bi-crystalline micro-pillars. Crystals 10, 411.

Chen, Y.P., Cai, Y.Y., Guo, J.P., Pan, E., 2017. Interfacial elastic fields of a 3D dislocation loop in anisotropic bimaterials of finite thickness crystal films. Mechanics of Materials 113, $1-18$.

Chen, Y.P., Zhang, X.Q., He, Y.M., Pan, E., 2019b. Elastic field by a dislocation loop in an anisotropic elastic half-space with general boundary conditions and its application in nanoindentation of single crystals. International Journal of Mechanical Sciences 160, 26-37.

Choi, S.T., Earmme, Y.Y., 2002a. Elastic study on singularities interacting with interfaces using alternating technique: Part I. anisotropic trimaterial. International Journal of Solids and Structures 39, 943-957.

Choi, S.T., Earmme, Y.Y., 2002b. Elastic study on singularities interacting with 
interfaces using alternating technique: Part II. isotropic trimaterial. International Journal of Solids and Structures 39, 1199-1211.

Chou, Y., Li, J., 1969. Theory of Dislocation Pileups, in: Proceedings of the Symposium on the Mathematical Theory of Dislocations. ASME Publication.

Chu, H., Pan, E., 2014. Elastic fields due to dislocation arrays in anisotropic bimaterials. International Journal of Solids and Structures 51, 1954-1961.

Chu, H.J., Pan, E., Han, X., Wang, J., Beyerlein, I.J., 2012. Elastic fields of dislocation loops in three-dimensional anisotropic bimaterials. Journal of the Mechanics and Physics of Solids 60, $418-431$.

Chu, H.J., Wang, J., Beyerlein, I.J., Pan, E., 2013. Dislocation models of interfacial shearing induced by an approaching lattice glide dislocation. International Journal of Plasticity 41, 1 - 13.

Cordero, Z.C., Knight, B.E., Schuh, C.A., 2016. Six decades of the HallPetch effect a survey of grain-size strengthening studies on pure metals. International Materials Reviews 61, 495-512.

Davoudi, K.M., Lucia, N., Vlassak, J.J., 2014. Bauschinger effect in thin metal films: Discrete dislocation dynamics study. Journal of Applied Physics 115, 013507.

Dewit, R., 2008. Elastic constants and thermal expansion averages of a non textured polycrystal. Journal of Mechanics of Materials and Structures 3, 195-212.

Dieter, G., 1986. Mechanical Metallurgy. McGraw-Hill Higher Education. 
Dodaran, M., Wang, J., Shamsaei, N., Shao, S., 2020. Investigating the interaction between persistent slip bands and surface hard coatings via crystal plasticity simulations. Crystals 10, 1012.

Eshelby, J.D., Frank, F.C., Nabarro, F.R.N., 1951. The equilibrium of linear arrays of dislocations. Philosophical Magazine 42, 351-364.

Eshelby, J.D., Read, W.T., Shockley, W., 1953. Anisotropic elasticity with applications to dislocation theory. Acta Metallurgica 1, 251-259.

Espinosa, H.D., Panico, M., Berbenni, S., Schwarz, K.W., 2006. Discrete dislocation dynamics simulations to interpret plasticity size and surface effects in freestanding FCC thin films. International Journal of Plasticity 22, $2091-2117$.

Espinosa, H.D., Prorok, B.C., Peng, B., 2004. Plasticity size effects in free-standing submicron polycrystalline FCC films subjected to pure tension. Journal of the Mechanics and Physics of Solids 52, $667-689$.

Gao, Y., Liu, Z.L., Zhuang, Z., 2010. The investigation of size effect for dislocation starvation mechanism under $\mathrm{Cu}$ single crystal micro-pillar compression. AIP Conference Proceedings 1233, 436-441.

Gemperlova, J., Saxl, I., 1968. Dislocations in an inhomogeneous anisotropic medium. Czechoslovak Journal of Physics B 18, 1085-1093.

Hall, E.O., 1951. The deformation and ageing of mild steel: III discussion of results. Proceedings of the Physical Society. Section B 64, 747-753.

Han, S., Eisenlohr, P., Crimp, M.A., 2018. Ecci based characterization of dislocation shear in polycrystalline arrays during heterogeneous deformation of commercially pure titanium. Materials Characterization 142, 504-514. 
Han, X., Ghoniem, N.M., 2005. Stress field and interaction forces of dislocations in anisotropic multilayer thin films. Philosophical Magazine 85, 1205-1225.

Haouala, S., Alizadeh, R., Bieler, T.R., Segurado, J., LLorca, J., 2020. Effect of slip transmission at grain boundaries in Al bicrystals. International Journal of Plasticity 126, 102600.

Hill, R., 1952. The elastic behaviour of a crystalline aggregate. Proceedings of the Physical Society. Section A 65, 349 - 354.

Idrissi, H., Bollinger, C., Boioli, F., Schryvers, D., Cordier, P., 2016. Lowtemperature plasticity of olivine revisited with in situ TEM nanomechanical testing. Science Advances 2.

Javaid, F., Xu, Y., Durst, K., 2020. Local analysis on dislocation structure and hardening during grain boundary pop-ins in tungsten. Journal of Materials Science $55,9597-9607$.

Jebahi, M., Cai, L., Abed-Meraim, F., 2020. Strain gradient crystal plasticity model based on generalized non-quadratic defect energy and uncoupled dissipation. International Journal of Plasticity 126, 102617.

Keller, C., Hug, E., Habraken, A.M., Duchene, L., 2012. Finite element analysis of the free surface effects on the mechanical behavior of thin nickel polycrystals. International Journal of Plasticity 29, 155 - 172.

Kheradmand, N., 2012. Grain boundary-dislocation interaction : A local investigation via micron-sized bicrystals. Ph.D. thesis. Universität des Saarlandes, Germany. 
Kirchlechner, C., Grosinger, W., Kapp, M.W., Imrich, P.J., Micha, J.S., Ulrich, O., Keckes, J., Dehm, G., Motz, C., 2012. Investigation of reversible plasticity in a micron-sized, single crystalline copper bending beam by X-ray $\mu$ laue diffraction. Philosophical Magazine 92, 3231-3242.

Kochmann, D.M., Le, K., 2008. Dislocation pile-ups in bicrystals within continuum dislocation theory. International Journal of Plasticity 24, 2125 - 2147.

Kondo, S., Mitsuma, T., Shibata, N., Ikuhara, Y., 2016. Direct observation of individual dislocation interaction processes with grain boundaries. Science Advances 2, E1501926.

Lee, T.C., Robertson, I.M., Birnbaum, H.K., 1990. Tem in situ deformation study of the interaction of lattice dislocations with grain boundaries in metals. Philosophical Magazine A 62, 131-153.

Leknitskii, S.G., 1963. Theory of Elasticity of an Anisotropic Elastic Body. HoldenDay.

Li, J.C.M., 1981. Dislocation sources, in: Ashby, M.F., Bullough, R., Hartley, C.S., Hirth, J.P. (Eds.), Dislocation Modelling of Physical Systems. Pergamon, pp. 498 $-518$.

Lim, H., Lee, M.G., Kim, J.H., Adams, B.L., Wagoner, R.H., 2016. Simulation of polycrystal deformation with grain and grain boundary effects. International Journal of Plasticity 81, 231-248.

Lin, F., Marteleur, M., Jacques, P.J., Delannay, L., 2018. Transmission of (332) [113] twins across grain boundaries in a metastable $\beta$-titanium alloy. International Journal of Plasticity 105, 195-210. 
Mompiou, F., Caillard, D., Legros, M., Mughrabi, H., 2012. In situ TEM observations of reverse dislocation motion upon unloading in tensile-deformed UFG aluminium. Acta Materialia 60, 3402-3414.

Nicola, L., Xiang, Y., Vlassak, J.J., Van der Giessen, E., Needleman, A., 2006. Plastic deformation of freestanding thin films: Experiments and modeling. Journal of the Mechanics and Physics of Solids 54, 2089 - 2110.

Niordson, C.F., Legarth, B.N., 2010. Strain gradient effects on cyclic plasticity. Journal of the Mechanics and Physics of Solids 58, 542-557.

Ovid'ko, I.A., Sheinerman, A.G., 2017. Grain boundary sliding, triple junction disclinations and strain hardening in ultrafine-grained and nanocrystalline metals. International Journal of Plasticity 96, $227-241$.

Pedersen, O.B., Brown, L.M., Stobbs, W.M., 1981. The bauschinger effect in copper. Acta Metallurgica 29, 1843-1850.

Petch, N.J., 1953. The cleavage strength of polycrystals. The Journal of the Iron and Steel Institute 174, 25-30.

Press, W.H., Teukolsky, S.A., Vetterling, W.T., Flannery, B.P., 2007. Numerical recipes: The Art of Scientific Computing. Third edition. Cambridge University Press.

Rajagopalan, J., Rentenberger, C., Karnthaler, H.P., Dehm, G., Saif, M.T.A., 2010. In situ tem study of microplasticity and bauschinger effect in nanocrystalline metals. Acta Materialia 58, 4772-4782. 
Richeton, T., Chen, X., Berbenni, S., 2020. Misorientation dependence of the grain boundary migration rate: role of elastic anisotropy. Philosophical Magazine 100, $2436-2457$.

Rys, M., Petryk, H., 2018. Gradient crystal plasticity models with a natural length scale in the hardening law. International Journal of Plasticity 111, 168-187.

Schäfer, F., Weiter, L., Marx, M., Motz, C., 2016. Quantifying the grain boundary resistance against slip transfer by experimental combination of geometric and stress approach using stage-I fatigue cracks. Philosophical Magazine 96, 3524-3551.

Schneider, T.M., George, E.P., Manescau, T.J., Zalezak, T., Hunfeld, J., Dlouhy, A., Eggeler, G., Laplanche, G., 2020. Analysis of strengthening due to grain boundaries and annealing twin boundaries in the CrCoNi medium-entropy alloy. International Journal of Plasticity 124, 155-169.

Schouwenaars, R., 2020. Self-energy, line tension and bow-out of grain boundary dislocation sources. International Journal of Plasticity 133, 102802.

Shen, Z., Wagoner, R.H., Clark, W.A.T., 1988. Dislocation and grain boundary interactions in metals. Acta Metallurgica 36, 3231-3242.

Stroh, A.N., 1958. Dislocations and cracks in anisotropic elasticity. Philosophical Magazine 3, 625646.

Suo, Z., 1990. Singularities, interfaces and cracks in dissimilar anisotropic media. Proceedings of the Royal Society A: Mathematical, Physical and Engineering Sciences $427,331358$.

Suresh, S., 1998. Fatigue of Materials. Cambridge University Press. 2 edition. 
Ting, T.C.T., 1992. Image singularities of Green's functions for anisotropic elastic half-spaces and bimaterials. The Quarterly Journal of Mechanics and Applied Mathematics 45, 119-139.

Tucker, M.O., Crocker, A.G., 1968. The plane boundary in anisotropic elasticity. Mechanics of Generalized Continua, 286-289.

Voskoboinikov, R.E., Chapman, S.J., Ockendon, J.R., Allwright, D.J., 2007. Continuum and discrete models of dislocation pile-ups. I. Pile-up at a lock. Journal of the Mechanics and Physics of Solids 55, 2007-2025.

Voyiadjis, G.Z., Song, Y., 2019. Strain gradient continuum plasticity theories: Theoretical, numerical and experimental investigations. International Journal of Plasticity $121,21-75$.

Wagoner, R.H., 1981. Calculating dislocation spacings in pile-ups at grain boundaries. Metallurgical Transactions A 12, 2015-2023.

Wang, H.Y., Wu, M.S., 2005. Green's function for an anisotropic film-substrate embedded with a screw dislocation. Engineering Analysis with Boundary Elements 29, 624-635.

Wang, H.Y., Wu, M.S., Fan, H., 2007. Image decomposition method for the analysis of a mixed dislocation in a general multilayer. International Journal of Solids and Structures 44, 1563-1581.

Wu, M.S., Wang, H.Y., 2007. Solutions for edge dislocation in anisotropic filmsubstrate system by the image method. Mathematics and Mechanics of Solids 12, $183-212$. 
Wulfinghoff, S., Forest, S., Böhlke, T., 2015. Strain gradient plasticity modeling of the cyclic behavior of laminate microstructures. Journal of the Mechanics and Physics of Solids 79, 1-20.

Xiao, X., Terentyev, D., Chen, Q., Yu, L., Chen, L., Bakaev, A., Duan, H., 2017. The depth dependent hardness of bicrystals with dislocation transmission through grain boundaries: A theoretical model. International Journal of Plasticity 90, 212 -230 .

Yuan, J., Huang, Y., Chen, W., Pan, E., Kang, G., 2019. Theory of dislocation loops in multilayered anisotropic solids with magneto-electro-elastic couplings. Journal of the Mechanics and Physics of Solids 125, 440 - 471.

Zhang, Y., Liu, J., Chu, H., Wang, J., 2016. Elastic fields of a core-spreading dislocation in anisotropic bimaterials. International Journal of Plasticity 81, 231248. 


\title{
Appendix A. List of notations used in the image decomposition method
}

\section{Superscripts and subscripts}

\author{
$i, j, k, l \quad$ subscripts of a vector or a tensor in the three dimensional Cartesian coordi- \\ nates, where $i, j, k, l=1,2$ and 3 \\ $s \quad$ symbol that denotes a single dislocation \\ $t \quad x_{1}-, x_{2}-$ and $x_{3}-$ components of the variable, which corresponds to $t=1,2$ \\ and 3 , respectively \\ $K \quad$ index of corresponding subproblem \\ $J \quad$ index of the image dislocation densities for each subproblem \\ $U \quad$ upper interface of each crystal \\ $L \quad$ lower interface of each crystal \\ $R \quad$ real part of the variable \\ I imaginary part of the variable \\ $\alpha \quad$ index of the eigenvalues with positive imaginary parts and the associated \\ eigenvectors of the Stroh sextic equation $(\alpha=1,2$ and 3$)$
}

\section{Variables}




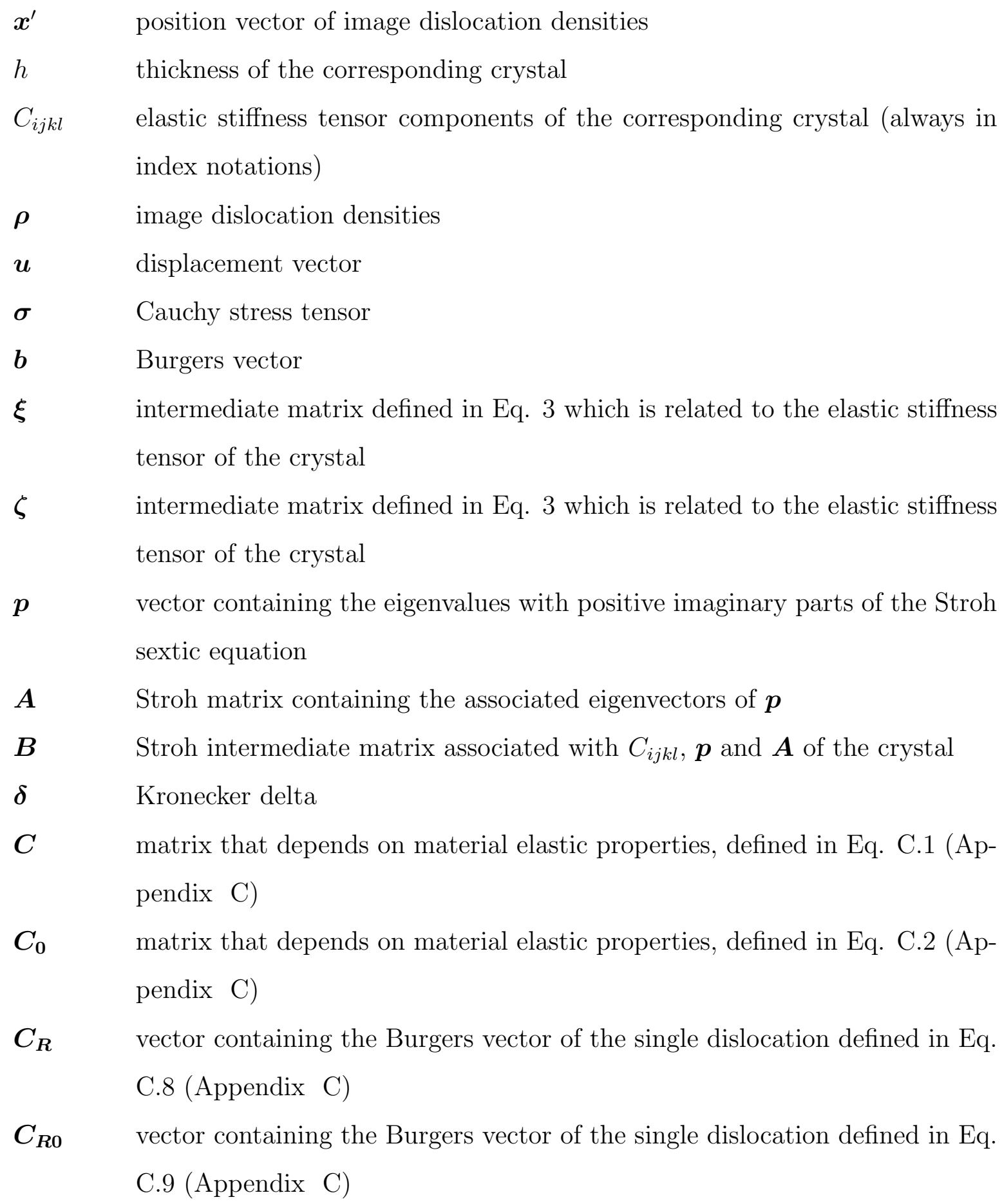




\section{Functions}

$P() \quad$ rational function of first-order polynomials defined in Eq. 4

$Q$ () rational function of second-order polynomials defined in Eq. 4

$H() \quad$ integral transform function defined in Eq. 7

I () integral transform function defined in Eq. 7

$\operatorname{sgn}() \quad$ sign function

$\delta() \quad$ Dirac delta function

$\boldsymbol{H}_{\mathbf{0}}$ matrix of integral operators for $\boldsymbol{\rho}$ defined in Eq. C.3 (Appendix C)

$\boldsymbol{C}_{\boldsymbol{H}}$ matrix of integral operators for $\boldsymbol{\rho}$ defined in Eq. C.4 (Appendix C)

$\boldsymbol{C}_{\boldsymbol{I}} \quad$ matrix of integral operators for $\boldsymbol{\rho}$ defined in Eq. C.5 (Appendix C)

$\boldsymbol{C}_{\boldsymbol{H 0}} \quad$ matrix of integral operators for $\boldsymbol{\rho}$ defined in Eq. C.6 (Appendix C)

$\boldsymbol{C}_{\boldsymbol{I 0}}$ matrix of integral operators for $\boldsymbol{\rho}$ defined in Eq. C.7 (Appendix C)

In our notation, the Einstein's convention over repeated indices is used when indices are underlined (see e.g. Eq. 1). Bold characters denote matrices or vectors.

\section{Appendix B. Mathematical method of Wang et al. (2007) for singularity problems in integral equations}

In Eq. 8, a singularity term is found with $2 \pi\left[\xi_{\underline{1} \underline{\underline{\alpha} i 2}}^{\underline{t} R} H(0,0)+\xi_{\underline{\underline{\alpha}} \underline{i} 2}^{\underline{I} I} I\left(0,0^{-}\right)\right]\left[\rho_{1 U}^{\underline{t}}\left(x_{1}\right)\right]$, in which:

$$
\begin{gathered}
2 \pi \xi_{\underline{1} \underline{\underline{\alpha} i 2}}^{\underline{t} R} H(0,0)\left[\rho_{1 U}^{\underline{t}}\left(x_{1}\right)\right]=2 \xi_{\underline{1} \underline{\underline{\alpha}} i 2}^{\underline{t} R} \int_{-\infty}^{\infty} P\left(x_{1}-x_{1}^{\prime}, 0,0\right) \rho_{1 U}^{\underline{t}}\left(x_{1}^{\prime}\right) d x_{1}^{\prime} \\
2 \pi \xi_{1 \underline{\alpha} i 2}^{\underline{t} I} I\left(0,0^{-}\right)\left[\rho_{1 U}^{\underline{t}}\left(x_{1}\right)\right]=2 \xi_{1 \underline{\underline{\alpha}} i 2}^{\underline{t} I} \int_{-\infty}^{\infty} Q\left(x_{1}-x_{1}^{\prime}, 0,0^{-}\right) \rho_{1}^{\underline{t}}\left(x_{1}^{\prime}\right) d x_{1}^{\prime}
\end{gathered}
$$


In the case where $a \rightarrow 0$ and $b \rightarrow 0$, the limiting values of the functions $P$ and $Q$ are given by (Wang et al., 2007):

$$
\begin{gathered}
\operatorname{Lim}_{a, b \rightarrow 0} P\left(x_{1}-x_{1}^{\prime}, a, b\right)=P\left(x_{1}-x_{1}^{\prime}, 0,0\right)=\frac{1}{x_{1}-x_{1}^{\prime}} \\
\operatorname{Lim}_{a, b \rightarrow 0} Q\left(x_{1}-x_{1}^{\prime}, a, b\right)=Q\left(x_{1}-x_{1}^{\prime}, 0,0\right)=\pi \operatorname{sgn}(b) \delta\left(x_{1}-x_{1}^{\prime}\right)
\end{gathered}
$$

The sign of the limit of $Q$ depends on its third argument $b$ which is especially important when limit value is evaluated at an infinitesimally small distance above and below an interface (Wang et al., 2007). Using the expression of Eq. B.3, Eq. B.1 can be written as:

$$
2 \pi \xi_{\underline{1} \underline{\underline{\alpha} i 2}}^{\underline{t} R} H(0,0)\left[\rho_{1 U}^{\underline{t}}\left(x_{1}\right)\right]=2 \xi_{\underline{1} \underline{\underline{\alpha} i 2}}^{\underline{t}} \int_{-\infty}^{\infty} \frac{\rho_{1 U}^{\underline{t}}\left(x_{1}^{\prime}\right)}{x_{1}-x_{1}^{\prime}} d x_{1}^{\prime}
$$

Hence, it can be seen that Eq. B.5 displays a singularity in the integral equation. In order to remove this singularity, Wang et al. (Wang and $\mathrm{Wu}, 2005$; Wu and Wang, 2007) have developed the technique of the $H$ and $I$ integral transforms. These $H$ and $I$ transforms can be used on $P$ and $Q$ functions or on themselves via residue calculus. In particular, using $H(0,0)$ on itself can be used to remove the singularity problem in Eq. B.5 as:

$$
H(0,0) H(0,0)=-I\left(0,0^{+}\right)
$$

Then, combining Eqs. 7 and B.4, the transform $I\left(0,0^{ \pm}\right)$on image dislocation densities can be calculated as:

$$
I\left(0,0^{ \pm}\right)\left[\rho\left(x_{1}\right)\right]=\operatorname{sgn}\left(0^{ \pm}\right) \rho\left(x_{1}\right)
$$


Thus, combining Eqs. B.6 and B.7, the singularity terms coming from $H(0,0)$ can be removed, and the image dislocation densities appear by themselves. However, when the transform $H(0,0)$ is used on all the terms, the product $H(0,0) I\left(0,0^{ \pm}\right)=$ $\operatorname{sgn}\left(0^{ \pm}\right) H(0,0)$ produce new singularity terms $(H(0,0)$ is directly applied on image dislocation densities themselves). In order to solve this problem, Wang et al. (2007) have developed a numerical method which is described below. Combining all the 18 boundary conditions, a system of nonlinear equations is formed with 18 Fredholm equations of second kind (i=1,2 and 3) with 18 unknowns $\boldsymbol{\rho}=\left[\rho_{1 U}^{t}, \rho_{1 L}^{t}, \rho_{2 U}^{t}, \rho_{2 L}^{t}, \rho_{3 U}^{t}, \rho_{3 L}^{t}\right]^{\mathrm{T}}$ as shown below:

$$
C \rho+C_{0} H_{0} \rho+C_{H} \rho+C_{I} \rho+C_{R}=0
$$

where $H_{0}, C_{H}, C_{I}$ are matrices of integral operators for the image dislocation densities $\boldsymbol{\rho}$ while $\boldsymbol{C}$ and $\boldsymbol{C}_{\mathbf{0}}$ are matrices whose coefficients only depend on material elastic properties. $\boldsymbol{C}_{\boldsymbol{R}}$ is a vector that contains the Burgers vector of the single dislocation. All the components of these matrices are reported in Appendix C. In Eq. B.8, the singularities $\boldsymbol{C}_{\mathbf{0}} \boldsymbol{H}_{\mathbf{0}} \boldsymbol{\rho}$ cannot be removed by applying $H(0,0)$ on all the terms because it would produce new singularities on the first term, as $\boldsymbol{C} \boldsymbol{H}_{\mathbf{0}} \boldsymbol{\rho}$. However, an implicit expression of the image dislocation densities can be obtained from solving the algebraic equation B. 8 as:

$$
\rho=-C^{-1}\left(C_{0} H_{0} \rho+C_{H} \rho+C_{I} \rho+C_{R}\right)
$$

Then, by substitution of Eq. B.9 in Eq. B.8, the final expression of the equations without singularity terms can be written as: 


$$
\begin{array}{r}
\left(C+C_{0} C^{-1} C_{0}\right) \rho+\left(C_{H}+C_{I}-C_{0} C^{-1} C_{H 0} H-C_{0} C^{-1} C_{I 0}\right) \rho \\
=-C_{R}+C_{0} C^{-1} C_{R 0}
\end{array}
$$

where the matricial expressions of integral operators $\boldsymbol{C}_{\boldsymbol{H} \mathbf{0}}, \boldsymbol{C}_{\boldsymbol{I 0}}$ and vector $\boldsymbol{C}_{\boldsymbol{R} \mathbf{0}}$ can be found in Appendix C. In the final expression (Eq. B.10), there is no term with integral operator $H(0,0)$ directly applied on the image dislocation densities $\boldsymbol{\rho}$, which proves that the singularity disappeared and can be easily used for integral calculations. 


\section{Appendix C. Full coefficient matrix of image decomposition method}

For one dislocation in a tri-material configuration with two free surfaces as presented in Figure 1 , the $18 \times 18$ matrices of coefficients $\boldsymbol{C}, \boldsymbol{C}_{\mathbf{0}}$, the $18 \times 18$ matrices of integral operators $\boldsymbol{H}_{\mathbf{0}}, \boldsymbol{C}_{\boldsymbol{H}}, \boldsymbol{C}_{\boldsymbol{I}}, \boldsymbol{C}_{\boldsymbol{H} \mathbf{0}}, \boldsymbol{C}_{\boldsymbol{I} \mathbf{0}}$, the $18 \times 1$ vectors in function of Burgers vector $\boldsymbol{C}_{\boldsymbol{R}}, \boldsymbol{C}_{\boldsymbol{R} \mathbf{0}}$ and the image dislocation densities vector $\boldsymbol{\rho}$ in Eqs. B.8, B.9 and B.10 are expressed in follows:

$$
\begin{aligned}
& \boldsymbol{C}=2 \pi\left[\begin{array}{cccccc}
-\xi_{1 \underline{\alpha} i 2}^{t R} & 0 & 0 & 0 & 0 & 0 \\
0 & 0 & 0 & 0 & 0 & -\xi_{3 \underline{\alpha} i 2}^{t R} \\
0 & -\xi_{1 \underline{\alpha} i 2}^{t R} & \xi_{2 \underline{\alpha} i 2}^{t R} & 0 & 0 & 0 \\
0 & \zeta_{1 \underline{\alpha} i 1}^{t I} & \zeta_{2 \underline{\alpha} i 1}^{t I} & 0 & 0 & 0 \\
0 & 0 & 0 & -\xi_{2 \underline{\alpha} i 2}^{t R} & \xi_{3 \underline{\alpha} i 2}^{t R} & 0 \\
0 & 0 & 0 & \zeta_{2 \underline{\alpha} i 1}^{t I} & \zeta_{3 \underline{\alpha} i 1}^{t I} & 0
\end{array}\right] \\
& \boldsymbol{C}_{\mathbf{0}}=2 \pi\left[\begin{array}{cccccc}
0 & 0 & 0 & 0 & 0 & 0 \\
0 & 0 & 0 & 0 & 0 & 0 \\
0 & 0 & 0 & 0 & 0 & 0 \\
0 & \zeta_{1 \underline{\alpha} i 1}^{t R} & -\zeta_{2 \underline{\alpha} i 1}^{t R} & 0 & 0 & 0 \\
0 & 0 & 0 & 0 & 0 & 0 \\
0 & 0 & 0 & \zeta_{\underline{2} \underline{\alpha} i 1}^{t R} & -\zeta_{3 \underline{\alpha} i 1}^{t R} & 0
\end{array}\right] \\
& \boldsymbol{H}_{\mathbf{0}}=2 \pi\left[\begin{array}{cccccc}
H(0,0) & 0 & 0 & 0 & 0 & 0 \\
0 & H(0,0) & 0 & 0 & 0 & 0 \\
0 & 0 & H(0,0) & 0 & 0 & 0 \\
0 & 0 & 0 & H(0,0) & 0 & 0 \\
0 & 0 & 0 & 0 & H(0,0) & 0 \\
0 & 0 & 0 & 0 & 0 & H(0,0)
\end{array}\right]
\end{aligned}
$$




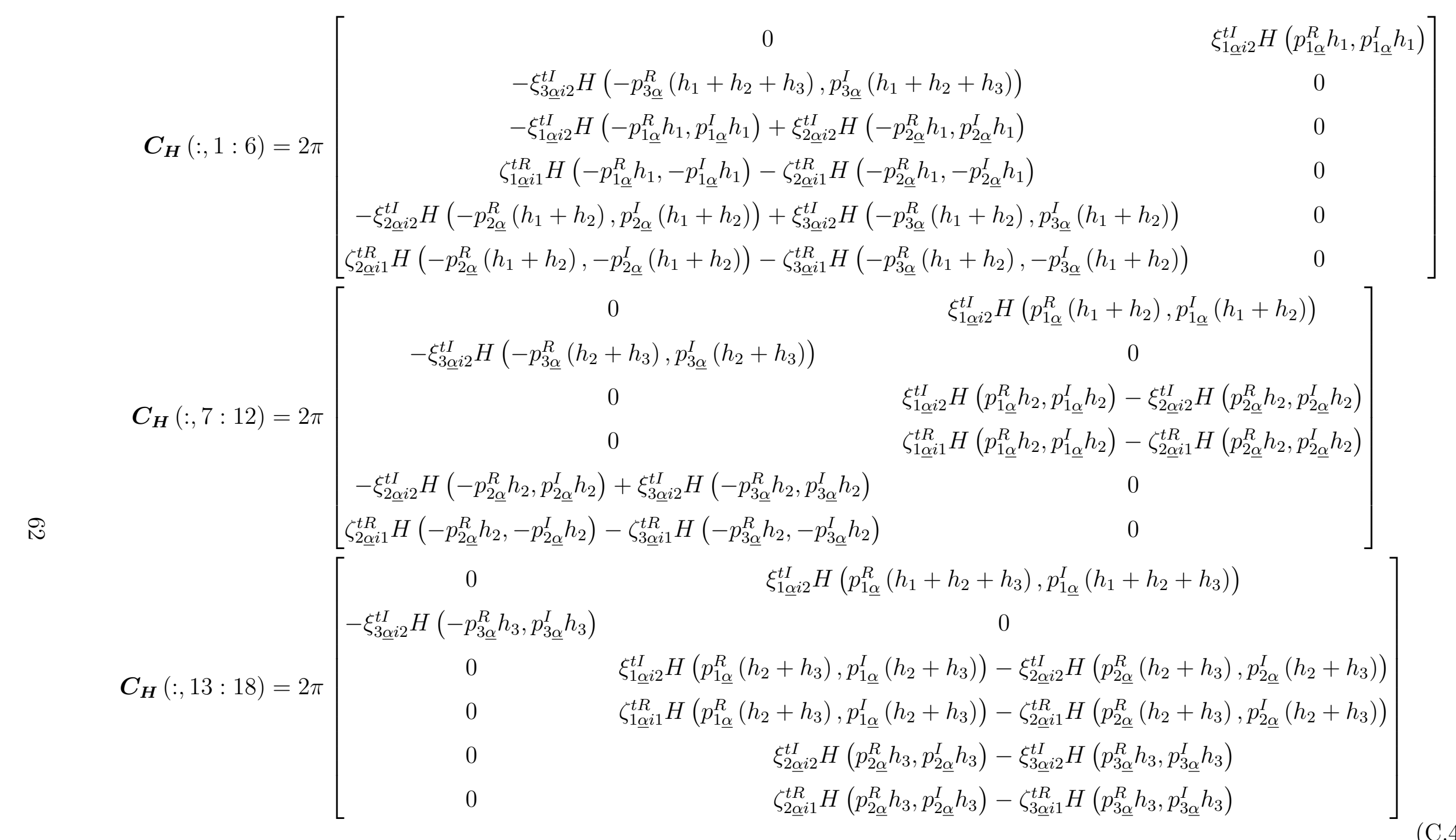




$$
\begin{aligned}
& 0 \\
& -\xi_{3 \underline{\alpha} i 2}^{t R} I\left(-p_{3 \underline{\alpha}}^{R}\left(h_{1}+h_{2}+h_{3}\right), p_{3 \underline{\alpha}}^{I}\left(h_{1}+h_{2}+h_{3}\right)\right) \\
& -\xi_{1 \underline{\alpha} i 2}^{t R} I\left(p_{1 \underline{\alpha}}^{R} h_{1}, p_{1 \underline{\alpha}}^{I} h_{1}\right) \\
& -\xi_{1 \underline{\alpha} i 2}^{t R} I\left(-p_{1 \underline{\alpha}}^{R} h_{1}, p_{1 \underline{\alpha}}^{I} h_{1}\right)+\xi_{2 \underline{\alpha} i 2}^{t R} I\left(-p_{2 \underline{\alpha}}^{R} h_{1}, p_{2 \underline{\alpha}}^{I} h_{1}\right) \quad 0 \\
& \zeta_{1 \underline{\alpha} i 1}^{t I} I\left(-p_{1 \underline{\alpha}}^{R} h_{1},-p_{1 \underline{\alpha}}^{I} h_{1}\right)-\zeta_{2 \underline{\alpha} i 1}^{t I} I\left(-p_{2 \underline{\alpha}}^{R} h_{1},-p_{2 \underline{\alpha}}^{I} h_{1}\right) \quad 0 \\
& -\xi_{2 \underline{\alpha} i 2}^{t R} I\left(-p_{2 \underline{\alpha}}^{R}\left(h_{1}+h_{2}\right), p_{2 \underline{\alpha}}^{I}\left(h_{1}+h_{2}\right)\right)+\xi_{3 \underline{\alpha} i 2}^{t R} I\left(-p_{3 \underline{\alpha}}^{R}\left(h_{1}+h_{2}\right), p_{3 \underline{\alpha}}^{I}\left(h_{1}+h_{2}\right)\right) \quad 0 \\
& \zeta_{2 \underline{\alpha} i 1}^{t I} I\left(-p_{2 \underline{\alpha}}^{R}\left(h_{1}+h_{2}\right),-p_{2 \underline{\alpha}}^{I}\left(h_{1}+h_{2}\right)\right)-\zeta_{3 \underline{\alpha} i 1}^{t I} I\left(-p_{3 \underline{\alpha}}^{R}\left(h_{1}+h_{2}\right),-p_{3 \underline{\alpha}}^{I}\left(h_{1}+h_{2}\right)\right) \\
& 0 \\
& -\xi_{3 \underline{\alpha} i 2}^{t R} I\left(-p_{3 \underline{\alpha}}^{R}\left(h_{2}+h_{3}\right), p_{3 \underline{\alpha}}^{I}\left(h_{2}+h_{3}\right)\right) \\
& -\xi_{1 \underline{\alpha} i 2}^{t R} I\left(p_{1 \underline{\alpha}}^{R}\left(h_{1}+h_{2}\right), p_{1 \underline{\alpha}}^{I}\left(h_{1}+h_{2}\right)\right) \\
& 0 \\
& 0 \\
& -\xi_{1 \underline{\alpha} i 2}^{t R} I\left(p_{1 \underline{\alpha}}^{R} h_{2}, p_{1 \underline{\alpha}}^{I} h_{2}\right)+\xi_{2 \underline{\alpha} i 2}^{t R} I\left(p_{2 \underline{\alpha}}^{R} h_{2}, p_{2 \underline{\alpha}}^{I} h_{2}\right) \\
& \boldsymbol{C}_{\boldsymbol{I}}(:, 7: 12)=2 \pi \\
& -\xi_{2 \underline{\alpha} i 2}^{t R} I\left(-p_{2 \underline{\alpha}}^{R} h_{2}, p_{2 \underline{\alpha}}^{I} h_{2}\right)+\xi_{3 \underline{\alpha} i 2}^{t R} I\left(-p_{3 \underline{\alpha}}^{R} h_{2}, p_{3 \underline{\alpha}}^{I} h_{2}\right) \\
& \zeta_{1 \underline{\alpha} i 1}^{t I} I\left(p_{1 \underline{\alpha}}^{R} h_{2}, p_{1 \underline{\alpha}}^{I} h_{2}\right)-\zeta_{2 \underline{\alpha} i 1}^{t I} I\left(p_{2 \underline{\alpha}}^{R} h_{2}, p_{2 \underline{\alpha}}^{I} h_{2}\right) \\
& \zeta_{2 \underline{\alpha} i 1}^{t I} I\left(-p_{2 \underline{\alpha}}^{R} h_{2},-p_{2 \underline{\alpha}}^{I} h_{2}\right)-\zeta_{3 \underline{\alpha} i 1}^{t I} I\left(-p_{3 \underline{\alpha}}^{R} h_{2},-p_{3 \underline{\alpha}}^{I} h_{2}\right) \\
& 0 \\
& \boldsymbol{C}_{\boldsymbol{I}}(:, 13: 18)=2 \pi\left[\begin{array}{c}
0 \\
-\xi_{3 \underline{\alpha} i 2}^{t R} I\left(-p_{3 \underline{\alpha}}^{R} h_{3}, p_{3 \underline{\alpha}}^{I} h_{3}\right) \\
0 \\
0 \\
0 \\
0
\end{array}\right. \\
& -\xi_{1 \underline{\alpha} i 2}^{t R} I\left(p_{1 \underline{\alpha}}^{R}\left(h_{1}+h_{2}+h_{3}\right), p_{1 \underline{\alpha}}^{I}\left(h_{1}+h_{2}+h_{3}\right)\right) \\
& 0 \\
& \begin{array}{c}
\left.-\xi_{1 \underline{\alpha} i 2}^{t R} I\left(p_{1 \underline{\alpha}}^{R}\left(h_{2}+h_{3}\right), p_{1 \underline{\alpha}}^{I}\left(h_{2}+h_{3}\right)\right)+\xi_{2 \underline{\alpha} i 2}^{t R} I\left(p_{2 \underline{\alpha}}^{R}\left(h_{2}+h_{3}\right), p_{2 \underline{\alpha}}^{I}\left(h_{2}+h_{3}\right)\right)\right) \\
\zeta_{1 \underline{\alpha} i 1}^{t I} I\left(p_{1 \underline{\alpha}}^{R}\left(h_{2}+h_{3}\right), p_{1 \underline{\alpha}}^{I}\left(h_{2}+h_{3}\right)\right)-\zeta_{2 \underline{\alpha} i 1}^{t I} I\left(p_{2 \underline{\alpha}}^{R}\left(h_{2}+h_{3}\right), p_{2 \underline{\alpha}}^{I}\left(h_{2}+h_{3}\right)\right) \\
-\xi_{2 \underline{\alpha} i 2}^{t R} I\left(p_{2 \underline{\alpha}}^{R} h_{3}, p_{2 \underline{\alpha}}^{I} h_{3}\right)+\xi_{3 \underline{\alpha} i 2}^{t R} I\left(p_{3 \underline{\alpha}}^{R} h_{3}, p_{3 \underline{\alpha}}^{I} h_{3}\right) \\
\zeta_{2 \underline{\alpha} i 1}^{t I} I\left(p_{2 \underline{\alpha}}^{R} h_{3}, p_{2 \underline{\alpha}}^{I} h_{3}\right)-\zeta_{3 \underline{\alpha} i 1}^{t I} I\left(p_{3 \underline{\alpha}}^{R} h_{3}, p_{3 \underline{\alpha}}^{I} h_{3}\right)
\end{array}
\end{aligned}
$$




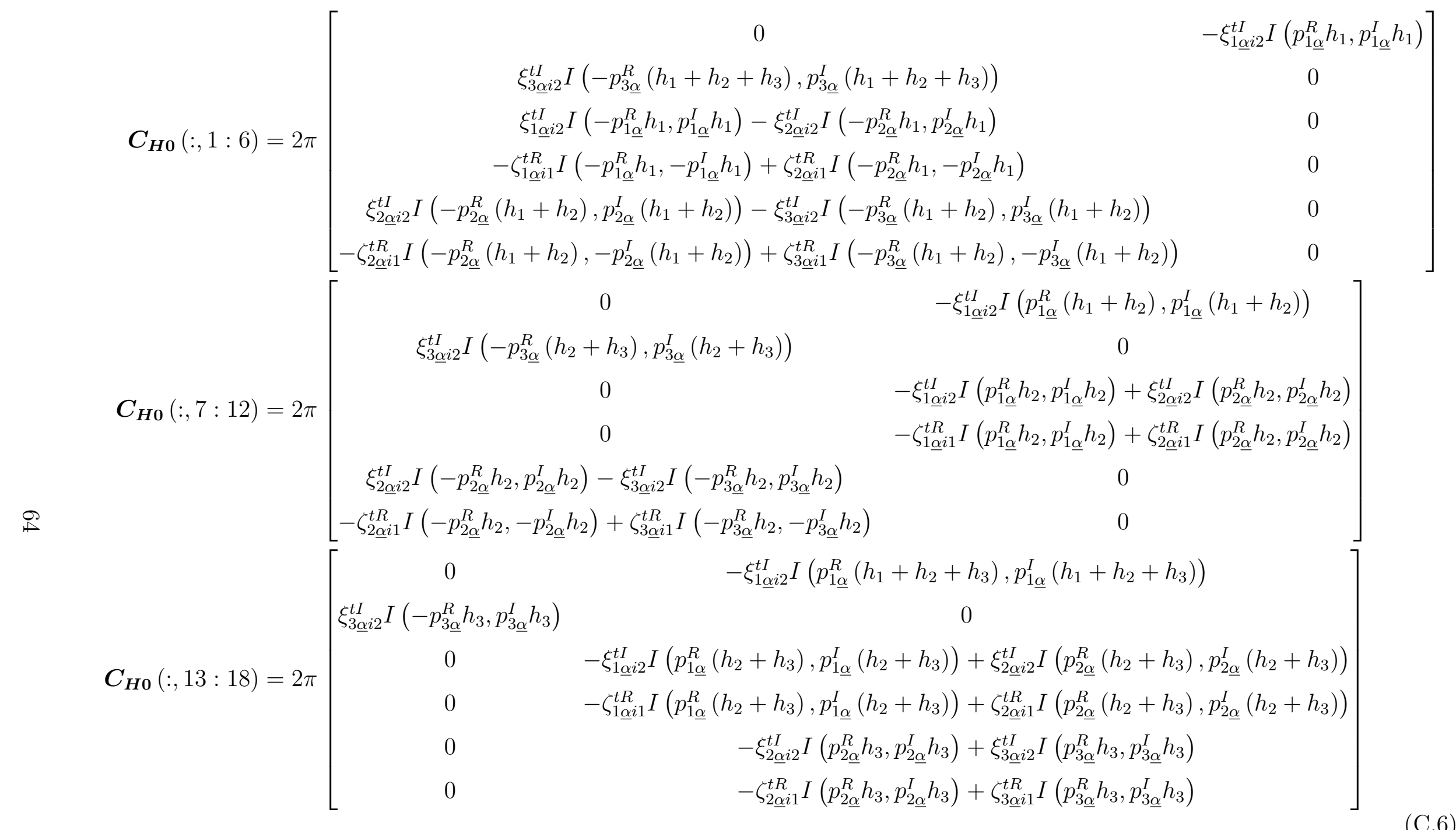


8 


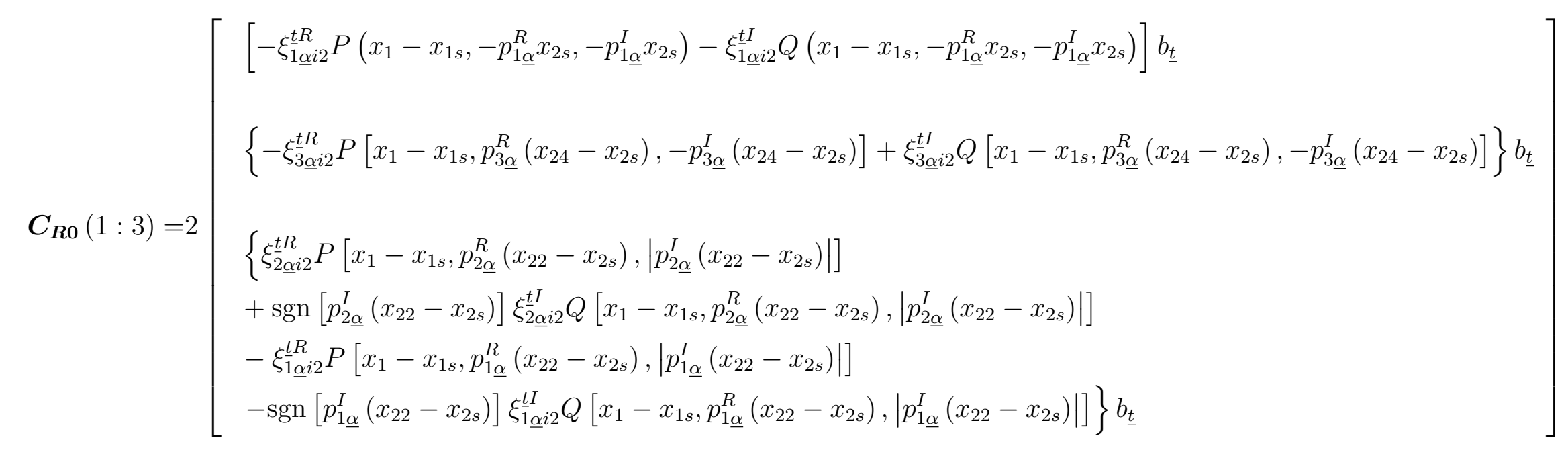

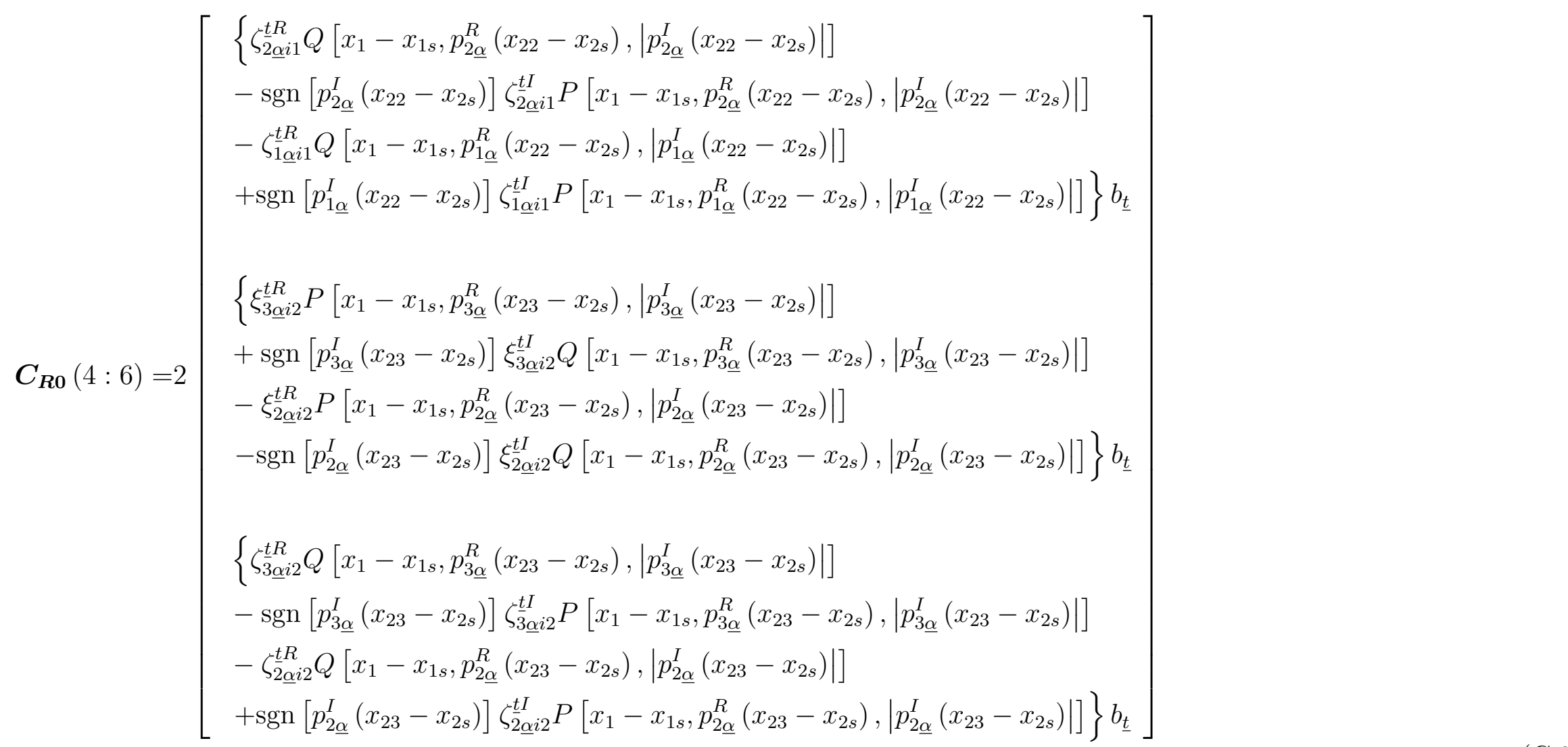


Each element in the previous matrices is a $3 \times 3$ matrix:

$$
\boldsymbol{\rho}=\left[\begin{array}{c}
\rho_{1 U}^{t} \\
\rho_{1 L}^{t} \\
\rho_{2 U}^{t} \\
\rho_{2 L}^{t} \\
\rho_{3 U}^{t} \\
\rho_{3 L}^{t}
\end{array}\right]
$$

$$
\begin{aligned}
& -\xi_{1 \underline{\alpha} 22}^{t R}=\left[\begin{array}{ccc}
-\xi_{1 \underline{\alpha} 12}^{1 R} & -\xi_{1 \underline{\alpha} 12}^{2 R} & -\xi_{1 \underline{1} 12}^{3 R} \\
-\xi_{1 \underline{\alpha} 22}^{R R} & -\xi_{1 \underline{\alpha} 22}^{2 R} & -\xi_{1 \underline{2} 22}^{3 R} \\
-\xi_{1 \underline{\alpha} 32}^{1 R} & -\xi_{1 \underline{\alpha} 32}^{2 R} & -\xi_{1 \underline{\alpha} 32}^{3 R}
\end{array}\right] \\
& H(0,0)=\left[\begin{array}{ccc}
H(0,0) & 0 & 0 \\
0 & H(0,0) & 0 \\
0 & 0 & H(0,0)
\end{array}\right] \\
& -\xi_{\underline{1} \underline{\alpha} 2}^{I I} I\left(p_{1 \underline{\alpha}}^{R} h_{1}, p_{1 \underline{\alpha}}^{I} h_{1}\right)=\left[\begin{array}{lll}
-\xi_{1 \underline{\alpha} 12}^{1 I} I\left(p_{1 \underline{\alpha}}^{R} h_{1}, p_{1 \underline{\alpha}}^{I} h_{1}\right) & -\xi_{1 \underline{\alpha} 12}^{2 I} I\left(p_{1 \underline{\alpha}}^{R} h_{1}, p_{1 \underline{\alpha}}^{I} h_{1}\right) & -\xi_{1 \underline{\alpha} 12}^{3 I} I\left(p_{1 \underline{\alpha}}^{R} h_{1}, p_{1 \underline{\alpha}}^{I} h_{1}\right) \\
-\xi_{1 \underline{\alpha} 22} I\left(p_{1 \underline{\alpha}}^{R} h_{1}, p_{1 \underline{\alpha}}^{I} h_{1}\right) & -\xi_{1 \underline{\alpha} 22}^{I I} I\left(p_{1 \underline{\alpha}}^{R} h_{1}, p_{1 \underline{\alpha}}^{I} h_{1}\right) & -\xi_{1 \underline{1} 22}^{3 I} I\left(p_{1 \underline{\alpha}}^{R} h_{1}, p_{1 \underline{\alpha}}^{I} h_{1}\right) \\
-\xi_{1 \underline{\alpha} 32}^{1 I} I\left(p_{1 \underline{\alpha}}^{R} h_{1}, p_{1 \underline{\alpha}}^{I} h_{1}\right) & -\xi_{1 \underline{\alpha} 32}^{2 I} I\left(p_{1 \underline{\alpha}}^{R} h_{1}, p_{1 \underline{\alpha}}^{I} h_{1}\right) & -\xi_{1 \underline{\alpha} 32}^{3 I} I\left(p_{1 \underline{\alpha}}^{R} h_{1}, p_{1 \underline{\alpha}}^{I} h_{1}\right)
\end{array}\right] \\
& {[0]=\left[\begin{array}{lll}
0 & 0 & 0 \\
0 & 0 & 0 \\
0 & 0 & 0
\end{array}\right]}
\end{aligned}
$$


Meanwhile, each element in the vector is a $3 \times 1$ vector, for example:

$$
\begin{aligned}
& {\left[-\xi_{1 \underline{\alpha} i 2}^{t R} Q\left(x_{1}-x_{1 s},-p_{1 \underline{\alpha}}^{R} x_{2 s},-p_{1 \underline{\alpha}}^{I} x_{2 s}\right)+\xi_{1 \underline{\alpha} i 2}^{t I} P\left(x_{1}-x_{1 s},-p_{1 \underline{\alpha}}^{R} x_{2 s},-p_{1 \underline{\alpha}}^{I} x_{2 s}\right)\right] b_{\underline{t}}}
\end{aligned}
$$

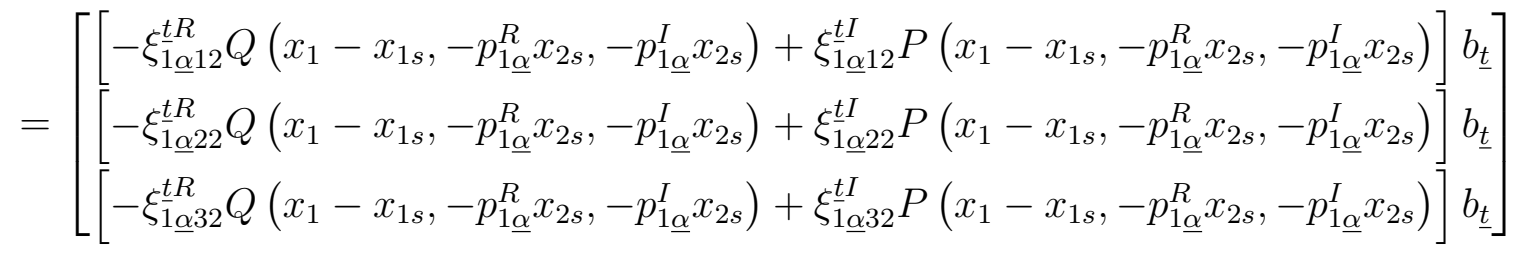

$$
\begin{aligned}
& \rho_{1 U}^{t}=\left[\begin{array}{c}
\rho_{1 U}^{1} \\
\rho_{1 U}^{2} \\
\rho_{1 U}^{3}
\end{array}\right]
\end{aligned}
$$

